\title{
CONNECTING THE GAMMA RAY BURST RATE AND THE COSMIC STAR FORMATION HISTORY: IMPLICATIONS FOR REIONIZATION AND GALAXY EVOLUTION
}

\author{
Brant E. Robertson ${ }^{1,2,3}$ and Richard S. ElLis ${ }^{1}$ \\ ${ }^{1}$ Astronomy Department, California Institute of Technology, MC 249-17, 1200 East California Boulevard, Pasadena, CA 91125, USA; brant@astro.caltech.edu \\ ${ }^{2}$ Steward Observatory, University of Arizona, 933 North Cherry Avenue, Tucson, AZ 85721, USA \\ Received 2011 September 5; accepted 2011 November 18; published 2011 December 19
}

\begin{abstract}
The contemporary discoveries of galaxies and gamma ray bursts (GRBs) at high redshift have supplied the first direct information on star formation when the universe was only a few hundred million years old. The probable origin of long duration GRBs in the deaths of massive stars would link the universal GRB rate to the redshift-dependent star formation rate (SFR) density, although exactly how is currently unknown. As the most distant GRBs and star-forming galaxies probe the reionization epoch, the potential reward of understanding the redshift-dependent ratio $\Psi(z)$ of the GRB rate to SFR is significant and includes addressing fundamental questions such as incompleteness in rest-frame UV surveys for determining the SFR at high redshift and time variations in the stellar initial mass function. Using an extensive sample of 112 GRBs above a fixed luminosity limit drawn from the Second Swift Burst Alert Telescope catalog and accounting for uncertainty in their redshift distribution by considering the contribution of "dark" GRBs, we compare the cumulative redshift distribution $N(<z)$ of GRBs with the star formation density $\dot{\rho}_{\star}(z)$ measured from UV-selected galaxies over $0<z<4$. Strong evolution (e.g., $\left.\Psi(z) \propto(1+z)^{1.5}\right)$ is disfavored (Kolmogorov-Smirnov test $P<0.07$ ). We show that more modest evolution (e.g., $\left.\Psi(z) \propto(1+z)^{0.5}\right)$ is consistent with the data $(P \approx 0.9)$ and can be readily explained if GRBs occur primarily in low-metallicity galaxies which are proportionally more numerous at earlier times. If such trends continue beyond $z \simeq 4$, we find that the discovery rate of distant GRBs implies an SFR density much higher than that inferred from UV-selected galaxies. While some previous studies of the GRB-SFR connection have concluded that GRB-inferred star formation at high redshift would be sufficient to maintain cosmic reionization over $6<z<9$ and reproduce the observed optical depth of Thomson scattering to the cosmic microwave background, we show that such a star formation history would overpredict the observed stellar mass density at $z>4$ measured from rest-frame optical surveys. The resolution of this important disagreement is currently unclear, and the GRB production rate at early times is likely more complex than a simple function of SFR and progenitor metallicity.
\end{abstract}

Key words: galaxies: evolution - gamma-ray burst: general

Online-only material: color figures

\section{INTRODUCTION}

The history of star formation in the universe is fundamental for determining the redshift-dependent properties of the galaxy population, the production of metals in the intergalactic medium (IGM), and the ionization state of cosmic gas. Observational probes of star formation at cosmological distances are therefore valuable tools for learning about the bulk properties of the universe and its contents (for a review, see Robertson et al. 2010).

The current frontier in this field concerns the observational determination of the comoving density of star formation $\dot{\rho}_{\star}(z)$ beyond a redshift $z \simeq 6$. This effort is key to understanding whether early galaxies were responsible for cosmic reionization as well as when this phase transition in the IGM occurred. Good progress has been made through efforts to identify restframe ultraviolet (UV)-selected galaxies in deep optical and near-infrared imaging out to $z \sim 8$ (e.g., Bouwens et al. 2007, 2010a, 2011b; Oesch et al. 2010; McLure et al. 2010). Recent spectroscopic campaigns have begun to confirm that many of these sources do indeed lie at $z \gtrsim 7$ (e.g., Iye et al. 2006; Stark et al. 2010; Lehnert et al. 2010; Fontana et al. 2010; Vanzella et al. 2011; Schenker et al. 2011; Pentericci et al. 2011; Ono et al. 2011). However, the direct detection of $z>6$ galaxies spanning

\footnotetext{
3 Hubble Fellow.
}

the full range of luminosities necessary to reliably deduce the integrated star formation rate (SFR) represents a challenge with current facilities. For example, the observationally inferred $\dot{\rho}_{\star}(z)$ depends on the adopted value of the poorly determined luminosity function faint end slope and the magnitude limit of the survey (McLure et al. 2010; Bouwens et al. 2011b).

As long-duration gamma ray bursts (GRBs) are thought to occur through the demise of very massive, possibly metal-poor stars (for a review, see Woosley \& Bloom 2006) and have been observationally connected to broad-line Type Ic supernovae (SNe Ic; e.g., Galama et al. 1998; Stanek et al. 2003; Hjorth et al. 2003), the rate of high-redshift GRBs of this class could provide a valuable, complementary estimate of $\dot{\rho}_{\star}(z)$ (e.g., Totani 1997; Wijers et al. 1998; Mao \& Mo 1998; Porciani \& Madau 2001; Bromm \& Loeb 2002; Chary et al. 2007). In particular, as luminous events, they could effectively probe the full extent of the star-forming galaxy population including low-mass systems undetected in the deepest optical and near-infrared surveys. The recent discovery of several $z>6$ long-duration GRBs (Kawai et al. 2006; Greiner et al. 2009; Tanvir et al. 2009; Salvaterra et al. 2009a; Cucchiara et al. 2011) makes this a particularly interesting avenue to explore.

The connection between the rate of GRBs (comoving $\left.\mathrm{Mpc}^{-3} \mathrm{yr}^{-1}\right)$ and the density of star formation $\left(M_{\odot} \mathrm{yr}^{-1} \mathrm{Mpc}^{-3}\right)$ can be coarsely parameterized by $\Psi(z)$, the ratio of the GRB rate to the SFR density $\dot{\rho}_{\star}$. The ratio $\Psi(z)$ could be inferred by 
comparing the cumulative redshift distribution $N(<z)$ of GRBs with the observed evolution of the SFR density $\dot{\rho}_{\star}(z)$ over the range of redshifts where $\dot{\rho}_{\star}(z)$ is well measured. Earlier work (Yüksel et al. 2008; Kistler et al. 2008, 2009; Wyithe et al. 2010) has suggested that this ratio increases with redshift in the sense that GRBs are more frequent for a given SFR density at earlier times. For instance, if such a redshift dependence is parameterized as a simple power law, $\Psi(z) \propto(1+z)^{\alpha}$, Kistler et al. (2009) find that $\alpha \sim 1.2$. Although the origin of such a redshift dependence remains unclear, if extrapolated to higher redshifts the abundance of GRBs at $z>7$ (Tanvir et al. 2009; Salvaterra et al. 2009a; Cucchiara et al. 2011) implies a density of star formation considerably higher $(\sim 6-7 \times$ larger $)$ than $\dot{\rho}_{\star}(z)$ measured from the abundance of distant UV-selected galaxies. In other words, the decline in the GRB rate at $z>4$ is considerably less rapid than the decrease in the SFR (e.g., Wanderman \& Piran 2010). This discrepancy has potentially important implications for the reionization history of the universe and particularly the contribution from early star-forming galaxies (e.g., Kistler et al. 2009; Wyithe et al. 2010).

Previous determinations of $\Psi(z)$ at low to intermediate redshift (e.g., Yüksel et al. 2008; Kistler et al. 2008, 2009; Ishida et al. 2011) have not accounted for the uncertainty in the GRB redshift distribution $N(<z)$ that arises from the socalled dark GRBs defined as those with absent or faint optical afterglows. Perley et al. (2009) have shown that dark GRBs likely span a wide range of redshifts and so they can be used to provide a valuable estimate of the uncertainty in the overall distribution, corresponding to approximately $25 \%$ in $N(<z)$ at fixed $z$; see their Figure 8 (see also, e.g., Greiner et al. 2011 and Krühler et al. 2011). In this work we use the recently released Second Swift Burst Alert Telescope (BAT) Catalog of GRBs (Sakamoto et al. 2011) to compile a comprehensive sample of 112 luminous $\left(L_{\text {iso }}>10^{51} \mathrm{erg} \mathrm{s}^{-1}\right)$ long-duration GRBs with known redshifts and upper limits (Butler et al. 2007, 2010; Fynbo et al. 2009; Sakamoto et al. 2011), accounting for the uncertainty in the overall distribution by including constraints provided for a representative subsample of dark GRBs (Perley et al. 2009; Greiner et al. 2011; Krühler et al. 2011).

Our goal is to revisit the redshift-dependent GRB rate to SFR ratio $\Psi(z)$ with this improved data set to determine carefully whether the ratio evolves and if so on what physical basis. A likely driver of such evolution is metallicity in the host galaxy population. To explore this possibility, we examine whether the evolution of the stellar mass-metallicity (e.g., Tremonti et al. 2004; Savaglio et al. 2005; Erb et al. 2006) and star-formation-stellar-mass (e.g., Drory \& Alvarez 2008) relations, when combined with the redshift-dependent stellar mass function (e.g., Drory et al. 2005), can explain the redshift scaling of $\Psi(z)$.

Our physical analysis of $\Psi(z)$ over $0<z<4$ enables us to calculate the high-redshift $(4<z<10)$ SFR density implied by the presence of distant GRBs and to compare this $\dot{\rho}_{\star}(z)$ with estimates drawn from rest-frame UV galaxy surveys. Following arguments developed in Robertson et al. (2010) we calculate the impact of this GRB-deduced SFR density on other indicators of the reionization history, including the optical depth of electron scattering to the cosmic microwave background and the stellar mass density that represents the integral of earlier activity. We show these additional constraints provide a critically important boundary on what is physically plausible in terms of the GRB-derived SFR at $z>6$ in addition to the likelihood of the various models we develop to explain the redshift dependence of $\Psi(z)$. We conclude by summarizing the issues that will need reckoning before the connection between the high-redshift GRB and SFRs is understood.

This paper is organized as follows. We construct a robust sample of GRBs above a fixed luminosity limit and discuss their redshift distribution and its uncertainties in Section 2. In Section 3, we compare the observed cumulative redshift distribution of GRBs over $0<z<4$ to the equivalent SFR density and its evolution. This comparison enables us to consider whether and how the GRB rate to SFR ratio $\Psi$ evolves. In Section 4, we interpret our results in the context of a hypothesis where GRBs primarily occur in low-metallicity galaxies. We compare predictions of this model with the observed GRB redshift distribution. In Section 5, we then use the observed GRB rate beyond $z \simeq 4$ to infer the SFR density under various assumed forms for $\Psi(z)$. We discuss the ramifications of our results in the context of other constraints on cosmic reionization in Section 6.

Throughout the paper we have assumed a standard flat $\Lambda \mathrm{CDM}$ cosmology $\left(\Omega_{m}=0.3, \Omega_{\Lambda}=0.7, h=0.7\right)$.

\section{THE REDSHIFT DISTRIBUTION OF LONG DURATION GRBs}

Our goal is to construct a robust sample of GRBs, observed down to a fixed luminosity limit, within a redshift range where a meaningful comparison can be made with an independently determined SFR density, $\dot{\rho}_{\star}(z)$, from rest-frame UV surveys of galaxies. In this way, the connection between the GRB rate and the SFR density, $\Psi$, and its possible evolution can be studied.

In a manner similar to Kistler et al. (2009), we can describe the observable number of GRBs within a redshift range $z_{a} \leqslant z \leqslant z_{b}$ as

$$
N\left(z_{a}, z_{b}\right)=K \int_{z_{a}}^{z_{b}} \dot{\rho}_{\star}(z) \Psi(z) \frac{d V}{d z} \frac{d z}{1+z},
$$

where $\dot{\rho}_{\star}(z)$ is the global SFR density, $\Psi$ is the number of GRBs per unit SFR, $d V / d z$ is the redshift-dependent volume element, and the factor $1 /(1+z)$ accounts for cosmological time dilation affecting the observed GRB rate. The constant $K$ is a factor that accounts for the efficiency of the GRB search (e.g., areal coverage, the survey flux limit, etc.), but its value is unimportant for our purposes. ${ }^{4}$ We can remove the dependence on $K$ by simply constructing the cumulative redshift of GRBs over the redshift range $0<z<z_{\max }$, normalized to $N\left(0, z_{\max }\right)$, as

$$
N\left(<z \mid z_{\max }\right)=\frac{N(0, z)}{N\left(0, z_{\max }\right)} .
$$

The product $\dot{\rho}_{\star}(z) \Psi(z)$ then sensibly determines the cumulative redshift distribution of GRBs. Given this normalization, we will also sometimes refer to the redshift-dependent fraction of star formation that can produce GRBs $\psi(z)$, which is related to the number of GRBs per unit SFR as $\Psi(z)=\Psi_{0} \psi(z)$. The constant $\Psi_{0}$ then encodes the number of GRBs formed per unit mass of stars. The value of $\Psi_{0}$ cannot be determined independently of the unknown $K$ in Equation (1), but the time or redshift when $\Psi_{0}$ is defined does matter for a model where $\psi(z)$ is calculated directly (see Section 4 below) rather than averaged over some

\footnotetext{
4 A clear concern is the possible multiplicative degeneracy between $K$ and $\Psi(z)$ in Equation (1), and that possible redshift dependence in, e.g., the follow-up efficiency could mimic an evolution of $\Psi(z)$. We note that Dai (2009) has found that the cumulative peak photon flux distribution of Swift GRBs with and without spectroscopic redshifts is similar. This result suggests that the follow-up efficiency does not strongly depend on redshift.
} 
redshift interval (cf. Equation (2)). In such a case, we take $\Psi_{0}$ to be defined relative to when $\psi(z)=1$.

\subsection{GRB Catalog}

To evaluate the possibility of redshift dependence in the GRB to SFR density $\Psi(z)$ through Equation (1), we require an observational sample to construct the cumulative redshift distribution calculated by Equation (2). The primary requirements are a well-understood completeness in the redshift determinations above some GRB isotropic-equivalent luminosity. The distribution $N\left(<z \mid z_{\max }\right)$ can be determined from catalogs of GRBs monitored with gamma-ray satellites (e.g., Swift; Gehrels et al. 2004) and followed up from the ground (e.g., Fynbo et al. 2009). For our base catalog of GRBs, we take the union of the samples presented in Butler et al. (2007, 2010), Perley et al. (2009), Sakamoto et al. (2011), Greiner et al. (2011), and Krühler et al. (2011). We include only GRBs occurring before the end of the Second Swift BAT GRB Catalog and prefer the most recent redshifts for GRBs where the samples disagree. This union provides a sample of 164 GRBs with known redshifts and redshift upper limits, but two GRBs (071112C and 060505) have incomplete fluence or burst duration measures and are discarded. The remaining 162 long duration GRBs with redshifts or redshift limits serve as our base GRB catalog.

To account for the incompleteness owing to the flux limit of the Swift survey, we follow Kistler et al. (2009) and construct a subsample of with isotropic-equivalent luminosities $L_{\text {iso }}>$ $10^{51} \mathrm{erg} \mathrm{s}^{-1}$. The luminosity is computed as

$$
L_{\text {iso }} \equiv \frac{E_{\text {iso }}}{t_{90} /(1+z)},
$$

where $E_{\text {iso }}$ is the isotropic-equivalent energy, $t_{90}$ is the burst duration containing from $5 \%$ to $95 \%$ of the total fluence, and the factor of $(1+z)$ accounts for cosmological time dilation (e.g., Kistler et al. 2009). For isotropic-equivalent energies, 129 GRB $E_{\text {iso }}$ values are taken from Butler et al. $(2007,2010)$ and 4 recent values $(060908,090926 \mathrm{~B}, 091018$, and 091029) from Sakamoto et al. (2011). Additionally, the isotropic energy values for 21 further GRBs are computed from the fluences reported by Butler et al. (2007, 2010). The isotropic energies from the eight remaining GRBs (060512, 090814A, 090904B, 090927, 091020, 091024, 091127, and 091208B) are calculated from the 15 to $150 \mathrm{keV}$ fluences reported by Sakamoto et al. (2011), but are possibly lower limits given the typical energy range of $1-10,000 \mathrm{keV}$ for defining isotropic-equivalent quantities (e.g., Amati et al. 2002). The burst durations $t_{90}$ are taken from Table 1 of Sakamoto et al. (2011), except for GRBs 050820A, 060218, and 090529A taken from Butler et al. (2007, 2010). For our catalog of 162 GRBs with redshifts and redshift limits, this culling provides $112 \mathrm{GRBs}$ with isotropic-equivalent luminosities $L_{\text {iso }}>10^{51} \mathrm{erg} \mathrm{s}^{-1}$ for our analysis. The redshifts and limits, isotropic equivalent energies and luminosities, and burst durations of the full sample of 162 GRBs compiled from the union of the Butler et al. (2007), Perley et al. (2009), Butler et al. (2010), Sakamoto et al. (2011), Greiner et al. (2011) and Krühler et al. (2011) catalogs are provided for convenience in Table 1 in the Appendix.

Since we will use the cumulative redshift distribution $N(<z)$ of this sample as the basis for our analysis, it is important to consider its uncertainties. While the Swift catalogs provide a valuable compilation of gamma-ray detections, the redshift determinations are clearly influenced by their optical observability.
The phenomenon of so-called dark GRBs with suppressed optical counterparts could influence whether the observed $N(<z)$ is representative of that for all long-duration GRBs. Perley et al. (2009) have considered this important issue by attempting to constrain the redshift distribution of dark GRBs through deep searches that successfully located faint optical and near-infrared counterparts. The Perley et al. (2009) work provides us with two redshifts and nine redshift upper limits for a subsample of dark GRBs in our catalog. Greiner et al. (2011) and Krühler et al. (2011) have pursued this effort in parallel and have provided three additional redshifts and one redshift upper limit for dark GRBs in our catalog. We assume that the subsamples of dark GRBs with redshift upper limits presented by Perley et al. (2009), Greiner et al. (2011), and Krühler et al. (2011) are representative of that class, and therefore optionally incorporate those limits to characterize the effects of possible incompleteness of the Swift sample with firm redshift determinations. We also note that while the luminosity limit for our sample was chosen to match Kistler et al. (2009), at redshifts above $z>4$ the Swift sample is incomplete for this limit. However, fully accounting for this incompleteness would only increase the relative number of GRBs at high redshifts. As our following results show, our sample's luminosity limit is therefore conservative.

\section{COMPARING GRB RATES TO THE COSMIC STAR FORMATION HISTORY}

As we have yet to develop physical intuition into the connection between the rate of GRBs and $\dot{\rho}_{\star}(z)$, we will begin by an empirical comparison of the cumulative GRB redshift distribution $N\left(<z \mid z_{\max }\right)$ constructed as described in Section 2.1 with the cumulative redshift distribution that would be expected given the observed SFR density $\dot{\rho}_{\star}(z)$ from rest-frame UV-selected sample and various forms for the redshift-dependent ratio $\Psi(z)$.

For $\dot{\rho}_{\star}(z)$ we use the results from Hopkins \& Beacom (2006) who gathered and standardized measures of the SFR density from the Hopkins (2004) compilation and observations by Wolf et al. (2003), Bouwens et al. (2003a, 2003b), Bunker et al. (2004), Ouchi et al. (2004), Arnouts et al. (2005), Le Floc'h et al. (2005), Pérez-González et al. (2005), Schiminovich et al. (2005), Bouwens \& Illingworth (2006), Hanish et al. (2006), and Thompson et al. (2006). Hopkins \& Beacom (2006) provide a piecewise-linear "Modified Salpeter A initial mass function (IMF)" model in their Table 2 that provides a good statistical fit to the available star formation density data. We limit our use of their fit to $z<4$ where the data are optimal. We note that $\dot{\rho}_{\star}(z)$ is relatively flat $\left[\dot{\rho}_{\star}(z) \propto(1+z)^{-0.26}\right]$ in the interval $1<z<4$ where most of the GRBs with $L_{\text {iso }}>10^{51} \mathrm{erg} \mathrm{s}^{-1}$ occur. This scaling means the use of Equation (2) is particularly accurate as the factor of $\sim 2$ in the normalization of $\dot{\rho}_{\star}(z)$ allowed by the $3 \sigma$ uncertainty in the Hopkins \& Beacom (2006) fit is circumvented (see also Section 4 below). We note that using the Hopkins \& Beacom (2006) results for the Baldry \& Glazebrook (2003) IMF instead of the Salpeter A IMF model (see, e.g., Hopkins \& Beacom 2008) has little effect on our conclusions.

Figure 1 shows the cumulative redshift distribution of observed GRBs (black histogram), normalized over the redshift range $0<z<4$. The gray-shaded region shows how the distribution shifts in the limiting cases of all dark GRBs occurring at $z=0$ or the upper redshift limits determined by Perley et al. (2009), Greiner et al. (2011), and Krühler et al. (2011). Figure 1 compares the observed GRB cumulative redshift distribution $N\left(<z \mid z_{\max }=4\right)$ for the case of three models for the redshift evolution of the GRB rate to SFR ratio $\Psi(z)$. 


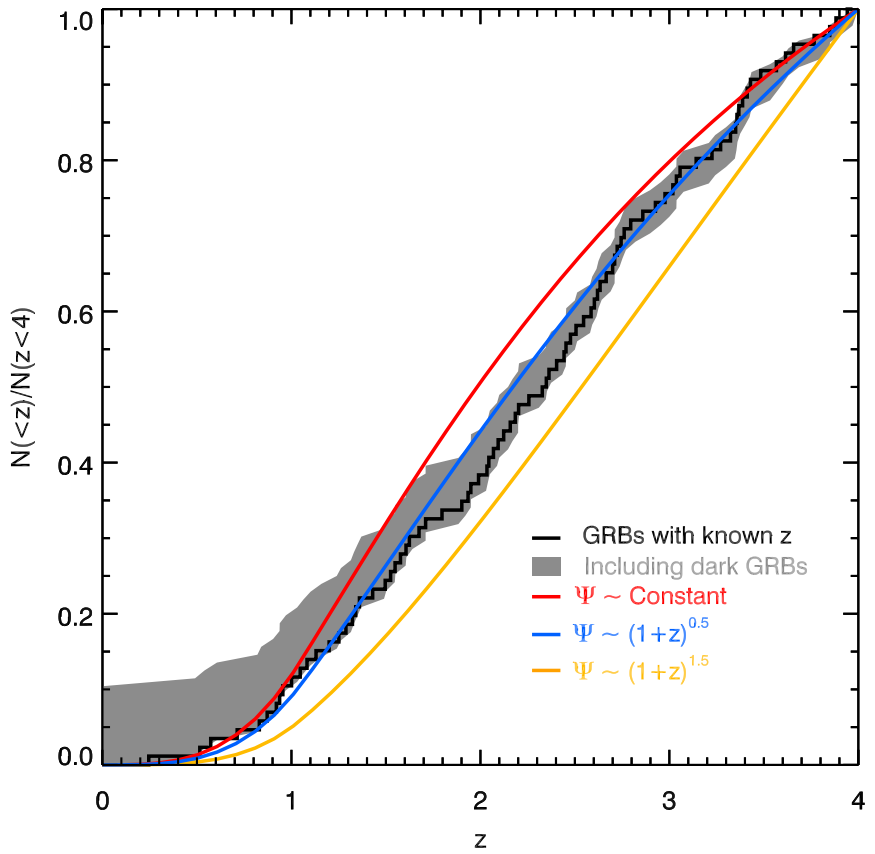

Figure 1. Cumulative redshift distribution of long-duration gamma-ray bursts (GRBs) at $z<4$ determined from the second Swift BAT catalog with isotropic equivalent luminosities $L_{\text {iso }}>10^{51} \mathrm{erg} \mathrm{s}^{-1}$ (black histogram, see the text for source of data). Incorporating the redshift constraints of dark GRBs shifts the distribution over the range indicated by the gray shaded area and provides an estimate of the intrinsic uncertainty in the observational determination of the distribution. Three simple power-law parameterizations of the ratio $\Psi(z)$ between the GRB and star formation rate densities are shown: a constant ratio with redshift (red line), $\Psi(z) \propto(1+z)^{0.5}$ (blue line), and $\Psi(z) \propto(1+z)^{1.5}$.

(A color version of this figure is available in the online journal.)

If the quantity $\Psi(z) \sim$ is constant (red line), the cumulative redshift distribution of GRBs increases rapidly at $z \sim 2-3$ in sync with the SFR density. If instead the GRB rate to SFR ratio evolves strongly with redshift over the epoch $z<4$ as, for instance, $\Psi(z) \propto(1+z)^{1.5}$ (orange line), then the GRB rate is shifted to higher redshifts and the cumulative distribution increases rapidly at $z>3$. A weaker redshift evolution $\left[\Psi(z) \propto(1+z)^{0.5}\right]$ better reproduces the cumulative GRB rate density (blue line). Given the relatively small sample, the data appear roughly consistent with each of these $\Psi(z)$ redshift scalings.

\subsection{Statistical Tests}

Given the integral distribution of observed GRB redshifts, and a parameterized model for predicting this distribution, we can perform statistical tests to check for consistency between the observed and model distributions, and calculate confidence intervals for the parameters of the model given the data. The null hypothesis that the observed GRB redshifts are consistent with a model distribution can be evaluated by the one-sample Kolmogorov-Smirnov (K-S) test, which calculates a " $P$-value" that corresponds to one minus the probability that the null hypothesis can be rejected. We employ this statistical test to determine a plausible range of values for the parameter $\alpha$ in a model where $\Psi(z) \propto(1+z)^{\alpha}$. We only use the K-S test to conservatively evaluate the relative agreement between the observed and model GRB redshift distributions. More formally, we can calculate the likelihood function for the parameter $\alpha$ given the observed data and assumed parameterized model using a Bayesian technique. For the case of $N$ independent samples

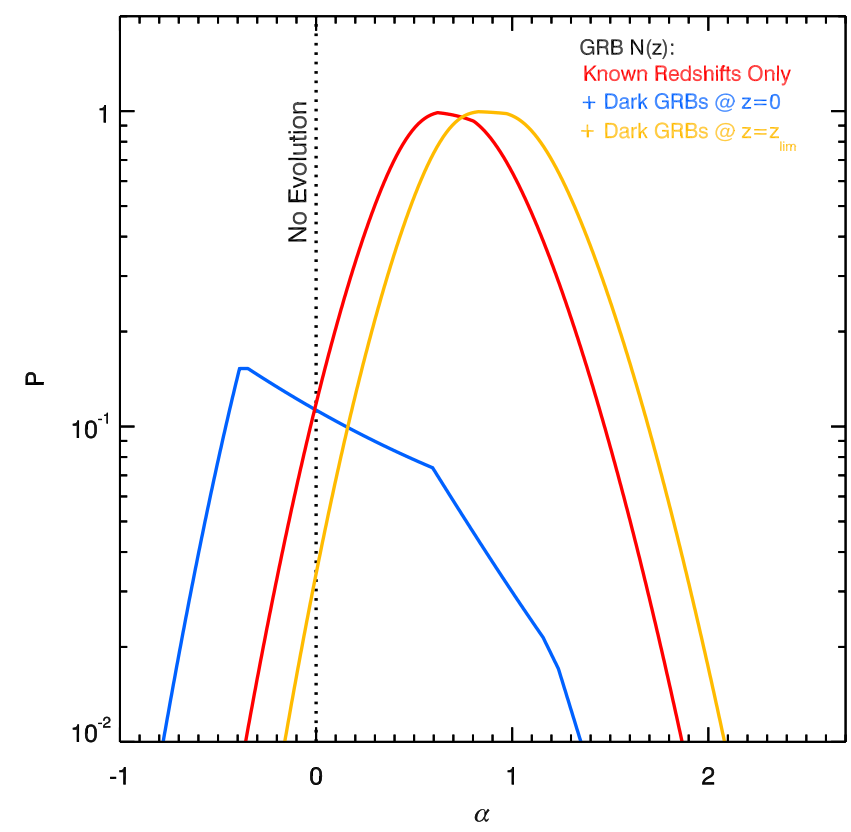

Figure 2. Results of a Kolmogorov-Smirnov test comparing the GRB cumulative redshift distribution $N\left(<z \mid z_{\max }=4\right)$ with model distributions calculated assuming the Hopkins \& Beacom (2006) model for the star formation rate density $\dot{\rho}_{\star}(z)$ with a redshift-dependent GRB rate to SFR ratio $\Psi(z) \propto(1+z)^{\alpha}$. Shown is the probability $P$ of consistency as a function of the power-law index $\alpha$. Comparing with sample with spectroscopic redshifts (red line), the $P>0.05$ interval is $-0.2<\alpha<1.5$ with a peak probability near $\alpha=0.5$. The Gaussian equivalent $2 \sigma$ confidence interval is $0.025<\alpha<1.5$ (see the text). If the dark GRB sample with redshift upper limits is assumed to be local $(z \approx 0)$, the $P>0.05$ interval shifts to $-0.7<\alpha<1$ with a median near $\alpha \approx 0$. If the dark GRBs lie at their maximum possible redshift the interval moves to $0<\alpha<1.8$ with a peak near $\alpha \approx 0.8$.

(A color version of this figure is available in the online journal.)

$\mathbf{z}=\left[z_{i}\right]$ from a redshift probability distribution $p(\mathbf{z} \mid \alpha)$ (e.g., the integrand of Equation (1)), the likelihood function of $\alpha$ can be calculated as

$$
\mathcal{L}(\alpha)=\prod_{i=0}^{N-1} p\left(z_{i} \mid \alpha\right)
$$

The posterior probability density of $\alpha$ given the observed data $z_{i}$ and parameterized model $\Psi$ can then be computed as

$$
p(\alpha \mid \mathbf{z}, \Psi)=\frac{p(\alpha \mid \Psi) \mathcal{L}(\alpha)}{\int d \alpha p(\alpha \mid \Psi) \mathcal{L}(\alpha)},
$$

where $p(\alpha \mid \Psi)$ is the prior probability of the parameter $\alpha$ given the model $\Psi$. We take this prior to be flat over a wide range of $\alpha$ such that it does not affect the shape of $p(\alpha \mid \mathbf{z}, \Psi)$. The confidence interval corresponding to a given Gaussian equivalent significance can then be calculated by integrating Equation (5) about the peak likelihood, and whenever an effective $\sigma$ is quoted it refers to the Bayesian confidence region.

Figure 2 shows the K-S test probability assuming $\Psi(z) \propto$ $(1+z)^{\alpha}$ for $-1<\alpha<2.5$. Comparing only with GRBs with spectroscopic redshifts (the solid black histogram in Figure 1), we find that the region where K-S $P>0.05$ contains powerlaw indices of $-0.2 \lesssim \alpha \lesssim 1.5$. The peak probability occurs for $\alpha \approx 0.5$. Computing the posterior probability $p(\alpha \mid \mathbf{z}, \Psi)$, we find that a constant $\Psi$ (i.e., no evolution) is marginally allowed at the $2 \sigma$ level. Including the dark GRB redshift constraints shifts the probability curve. If all dark GRBs are local, then the K-S test region $P>0.05$ contains power-law indices 
$-0.7<\alpha<1.0$ (with a median probability near $\alpha=0$ corresponding to no evolution). Instead, if all dark GRBs are at their maximum possible redshift then within $2 \sigma$ power-law indices $0.3<\alpha<1.7$ are favored. While the constraining power of the current sample is not particularly stringent, further monitoring of GRBs should soon definitively rule-out a $\Psi(z)$ that is constant or declines with redshift. We discuss the implications of this constraint further in Section 5.

In summary, we find that the distribution of GRBs with spectroscopic redshifts is consistent with only moderate variation of $\Psi(z)$ over $0 \lesssim z \lesssim 4$ and that there is overall only weak evidence for evolution ( $\sim 2 \sigma$ confidence). Compared to previous studies (e.g., Kistler et al. 2009) the results are consistent at the $\sim 2 \sigma$ level, but we infer a weaker redshift dependence owing to the fractionally increased number of GRBs at $z \lesssim 2$ in our compiled GRB sample. The additional uncertainty arising from including constraints from dark GRBs is important to include. If dark GRBs occur at their maximum allowed redshifts the distribution is more heavily weighted toward higher redshifts and more strongly indicates a possible redshift dependence to $\Psi(z)$.

Clearly, to make progress it would be helpful to have a physical basis for evolution in $\Psi(z)$. The most likely hypothesis links the GRB production rate with the metallicity of the underlying stellar population (e.g., Woosley \& Bloom 2006). Since the typical metallicity of galaxies at fixed stellar mass declines with redshift (e.g., Tremonti et al. 2004; Savaglio et al. 2005; Erb et al. 2006) this hypothesis provides a natural basis for an increase in $\Psi(z)$.

\section{MODELING THE REDSHIFT-DEPENDENT GRB TO SFR RATIO WITH METALLICITY EVOLUTION}

We now consider a physical scenario where the cosmic GRB rate is enhanced in low metallicity galaxies and develop a method for calculating the fraction of the SFR density occurring below a characteristic metallicity $12+\log [\mathrm{O} / \mathrm{H}]_{\text {crit }}$ above which GRB production is suppressed. Using the same statistical comparisons employed in Section 3, we can evaluate whether the observed GRB rate is consistent with such a picture and, if so, what values of $12+\log [\mathrm{O} / \mathrm{H}]_{\text {crit }}$ are favored.

A variety of theoretical pictures for the origin of long duration GRBs (e.g., Woosley 1993) suggest that the GRB rate may be connected to the metallicity of their progenitor stellar population (for reviews, see Mészáros 2006; Woosley \& Bloom 2006). GRBs may require the retention of significant angular momentum after collapse, which limits the amount of mass loss prior to collapse (e.g., Fryer et al. 1999; MacFadyen et al. 2001). The lower opacity and mass loss rates of low-metallicity stars ease this requirement and provide a credible rationale for preferring low-metallicity GRB hosts (Hirschi et al. 2005; Yoon \& Langer 2005; Woosley \& Heger 2006). Moreover, host galaxies of long duration GRBs are often observed to be metal poor and have low luminosities, both in the nearby universe (e.g., Prochaska et al. 2004; Sollerman et al. 2005; Modjaz et al. 2006; Stanek et al. 2006; Wiersema et al. 2007) and at cosmological distances (e.g., Fruchter et al. 1999, 2006; Le Floc'h et al. 2003; Fynbo et al. 2003; Savaglio et al. 2009).

The quantitative details of the metallicity connection are still debated (for a good discussion, see Modjaz 2011). For instance, by exploiting the connection between SNe Ic displaying broad lines and GRBs (e.g., Galama et al. 1998; Stanek et al. 2003; Hjorth et al. 2003), Modjaz et al. (2008) found that only low-metallicity $(12+\log [\mathrm{O} / \mathrm{H}]<8.7$ on the Kobulnicky \& Kewley 2004 scale, converted following Kewley \& Ellison
2008) galaxies have GRBs that fade into SNe Ic, whereas host galaxies with metallicities above this ceiling have GRB-free $\mathrm{SNe}$ Ic. However, there are regions within GRB host galaxies known with higher metallicities (Levesque et al. 2010b), and the radio-relativistic SN 2009bb (Soderberg et al. 2010) occurred in a high-metallicity region of its host galaxy (Levesque et al. 2010c). Han et al. (2010) and Levesque et al. (2010a) find that GRB host galaxies lie below the Tremonti et al. (2004) stellar-mass-metallicity relation, whereas Savaglio et al. (2009) report that the properties of GRB hosts do not clearly differ from normal star-forming galaxies. Mannucci et al. (2011) find that GRB hosts are offset from the mass-metallicity relation owing to their higher SFRs than average at fixed stellar mass, but remain within the "fundamental metallicity relation" between SFR, stellar mass, and metallicity (Mannucci et al. 2010).

Given this controversy, we considered it appropriate to construct a model to calculate $\Psi(z)$ in the case where GRBs preferentially occur in host galaxies with metallicities below some characteristic $12+\log [\mathrm{O} / \mathrm{H}]_{\text {crit }}$. Kocevski et al. (2009) combined the Modjaz et al. (2008) empirical host galaxy metallicity ceiling for GRBs with the redshift evolution of the stellar-mass-metallicity relation (Savaglio et al. 2005), the stellar-mass-star-formation rate relation evolution (Drory \& Alvarez 2008), and the stellar mass function evolution (Drory et al. 2005) to calculate the redshift-dependent characteristic mass of GRB host galaxies. Below, we extend the Kocevski et al. (2009) formalism to model the redshift dependence of $\Psi(z)$ and allow for a variable metallicity ceiling $12+\log [\mathrm{O} / \mathrm{H}]_{\text {crit }}$. We note that a similar approach has been adopted by Langer \& Norman (2006), Salvaterra \& Chincarini (2007), Salvaterra et al. (2009b), Butler et al. (2010) and Virgili et al. (2011). Analyses that used the Langer \& Norman (2006) calculation utilized the fraction of stellar mass at metallicities below $12+\log [\mathrm{O} / \mathrm{H}]_{\text {crit }}$ to determine the redshift dependence of $\Psi(z)$, whereas we prefer to extend the Kocevski et al. (2009) model to find $\Psi(z)$ from the fraction of star formation occurring at metallicities below $12+\log [\mathrm{O} / \mathrm{H}]_{\text {crit }}$.

\subsection{A Model for $\Psi(z)$ from Metallicity Evolution}

Given the relation between stellar mass and metallicity (e.g., Tremonti et al. 2004) a metallicity ceiling $12+\log [\mathrm{O} / \mathrm{H}]_{\text {crit }}$ for GRB host galaxies would imply a critical galaxy stellar mass $M_{\star, \text { crit }}$ above which GRB production is suppressed. The redshiftdependent stellar-mass-metallicity relation can be parameterized as (Savaglio et al. 2005, on the Kobulnicky \& Kewley 2004 scale)

$$
\begin{aligned}
12+\log [\mathrm{O} / \mathrm{H}]= & -7.5903+2.5315 \log M_{\star} \\
& -0.09649 \log ^{2} M_{\star} \\
& +5.1733 \log t_{u}-0.3944 \log ^{2} t_{u} \\
& -0.403 \log t_{u} \log M_{\star},
\end{aligned}
$$

where $t_{u}$ is the age of the universe at redshift $z$ in Gyr and $M_{\star}$ is the galaxy stellar mass in solar masses. Equation (6) then can be used to connect a given metallicity ceiling $12+\log [\mathrm{O} / \mathrm{H}]_{\text {crit }}$ to a critical galaxy mass $M_{\star, \text { crit }}(z)$ for GRB production.

We now introduce the fraction of star formation occurring in galaxies with metallicities lower than $12+\log [\mathrm{O} / \mathrm{H}]_{\text {crit }}$, which can be expressed as

$$
\psi(z)=\frac{\int_{0}^{M_{\star, \text { riti }}(z)} \operatorname{SFR}(M, z) \Phi_{\star}(M, z) d M}{\int_{0}^{\infty} \operatorname{SFR}(M, z) \Phi_{\star}(M, z) d M},
$$


where $\operatorname{SFR}(M, z)$ is the $\operatorname{SFR}$-stellar-mass relation and $\Phi_{\star}(M, z)$ is the galaxy stellar mass function. Drory \& Alvarez (2008) parameterize the observed SFR-stellar-mass relation as

$$
\operatorname{SFR}(M, z)=\operatorname{SFR}_{0}\left(\frac{M}{M_{0}}\right)^{\beta} \exp \left(-\frac{M}{M_{0}}\right),
$$

where $\beta \approx 0.5$ and the parameters $\mathrm{SFR}_{0}$ and $M_{0}$ evolve with redshift as

$$
\begin{gathered}
\operatorname{SFR}_{0}(z)=3.01(1+z)^{3.03} M_{\odot} \mathrm{yr}^{-1} \\
M_{0}(z)=2.7 \times 10^{10}(1+z)^{2.1} M_{\odot} .
\end{gathered}
$$

The stellar mass function also evolves with redshift (e.g., Drory et al. 2005). Taking a Schechter (1976) model for the galaxy stellar mass

$$
\Phi_{\star}(M, z)=\phi_{\star}\left(\frac{M}{M_{1}}\right)^{\gamma} \exp \left(-\frac{M}{M_{1}}\right) \frac{d M}{M_{1}},
$$

Drory \& Alvarez (2008) model the redshift dependence of the observed galaxy stellar mass function through the parameters

$$
\begin{gathered}
\phi_{\star}(z) \approx 0.003(1+z)^{-1.07} \mathrm{Mpc}^{-3} \mathrm{dex}^{-1} \\
\log \left[M_{1} / M_{\odot}\right](z) \approx 11.35-0.22 \ln (1+z) \\
\gamma(z) \approx-1.3 .
\end{gathered}
$$

By combining Equations (6) and (8)-(14) the redshiftdependent ratio of the GRB rate to the SFR $\Psi(z)$ can be estimated by evaluating Equation (5). With $\Psi(z)$ in hand, a model for the cumulative redshift distribution of GRBs can be calculated using Equations (1) and (2) once a model of the SFR density $\dot{\rho}_{\star}(z)$ is adopted. For a critical host galaxy metallicity $12+\log [\mathrm{O} / \mathrm{H}]_{\text {crit }}$ above which GRBs are suppressed, we can use the GRB data to inform us as to what metallicity ceilings are plausible. The redshift dependence of $\Psi(z)$ will clearly depend on the value of $12+\log [\mathrm{O} / \mathrm{H}]_{\text {crit }}$ since the fraction of star formation occurring at metallicities below $12+\log [\mathrm{O} / \mathrm{H}]_{\text {crit }}$ will vary with redshift owing to the evolution of the mass-metallicity relation, the mass-SFR relation, and the stellar mass function. For simplicity in this model, GRBs are prevented from occurring above $12+\log [\mathrm{O} / \mathrm{H}]_{\text {crit }}$, but as noted above in Section 4 GRBs do occur in metal rich host galaxies. The suppression in metal rich galaxies should therefore be understood to be an incomplete, coarse model to indicate the potential preference for GRBs to occur in metal-poor systems.

Figure 3 shows the cumulative GRB redshift distribution $N\left(<z \mid z_{\max }=4\right)$ resulting from Equation (7) with the adoption of the Hopkins \& Beacom (2006) SFR density model, for three choices for the critical metallicity $12+\log [\mathrm{O} / \mathrm{H}]_{\text {crit }}$. One model adopts a large $12+\log [\mathrm{O} / \mathrm{H}]_{\text {crit }}=9$ (red line), similar to the metallicity of the host of GRB 020819 (Levesque et al. 2010b). In this case, essentially all star formation occurs in hosts with metallicities below $12+\log [\mathrm{O} / \mathrm{H}]_{\text {crit }}$ and $\Psi$ is roughly constant with redshift as the GRB rate and SFR density trace one another. The large $12+\log [\mathrm{O} / \mathrm{H}]_{\text {crit }}$ model therefore closely tracks the $\alpha=0$ model shown in Figure 1 . An intermediate model adopts the value of $12+\log [\mathrm{O} / \mathrm{H}]_{\text {crit }} \approx 8.7$ from Modjaz et al. (2008),

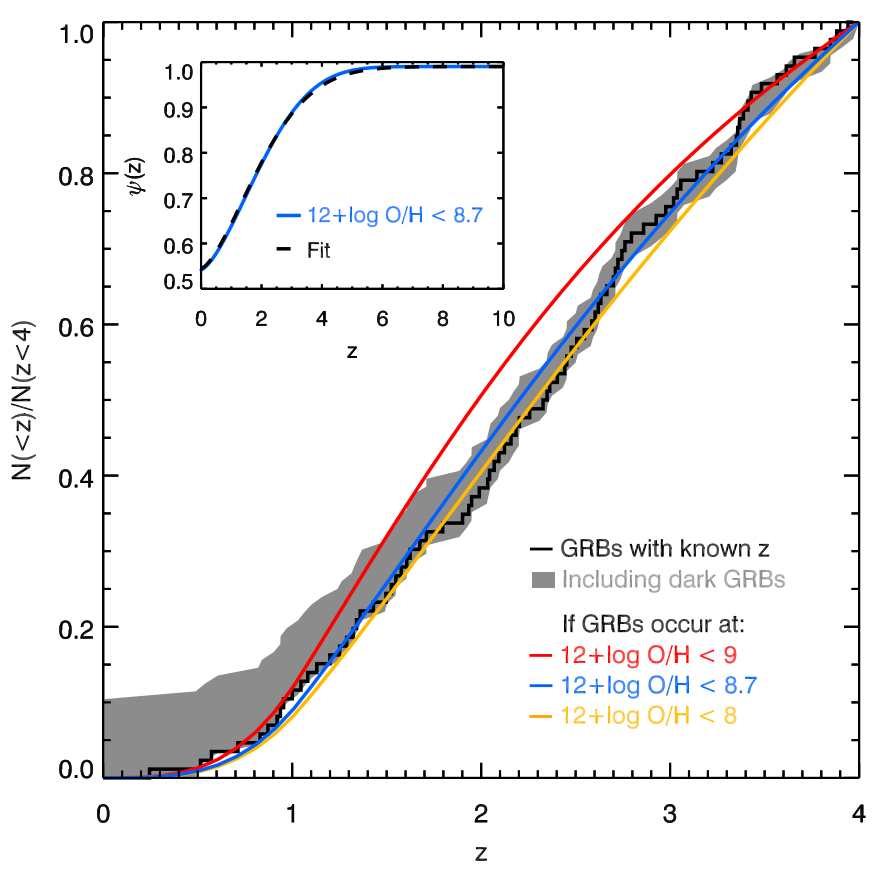

Figure 3. Cumulative redshift distribution of gamma-ray bursts at $z<4$ and metallicity-evolution models for the GRB rate to star formation rate ratio $\Psi(z)$. The black histogram and gray area indicate the cumulative distribution of GRBs with firm redshifts and the uncertainty owing to dark GRBs (see Figure 1 caption). Also shown are three models of the ratio $\Psi(z)$ where GRB production is suppressed in galaxies with metallicities above a ceiling $12+\log [\mathrm{O} / \mathrm{H}]_{\text {crit }}$, determined by model presented in Section 4: a high $12+\log [\mathrm{O} / \mathrm{H}]_{\text {crit }}=9$ (red line), the $12+\log [\mathrm{O} / \mathrm{H}]_{\text {crit }} \approx 8.7$ ceiling found by Modjaz et al. (2008, blue line), and a illustrative low ceiling $12+\log [\mathrm{O} / \mathrm{H}]_{\text {crit }}=8$ (orange line). The inset shows the redshift-dependence $\psi(z)$ corresponding to the model with $12+\log [\mathrm{O} / \mathrm{H}]_{\text {crit }} \approx 8.7$, along with a parameterized fit (see Equation (15)).

(A color version of this figure is available in the online journal.)

shown as the blue line in Figure 3. Star formation occurring in galaxies with metallicities below the Modjaz et al. (2008) $12+\log [\mathrm{O} / \mathrm{H}]_{\text {crit }}$ tracks the GRB rate with surprising fidelity, and for convenience we show the corresponding $\Psi(z)$ in the Figure 3 inset and provide a parameterized fit to this $\Psi(z)$ model as

$$
\Psi_{\text {fit }}(z)=0.5454+(1-0.5454) \times[\operatorname{erf}(0.324675 z)]^{1.45}
$$

(dashed black line in Figure 3 inset) that recovers the computed $\psi(z)$ to within $1 \%$ at $0<z<10$. Normalized over the redshift range $0<z<4$, this intermediate $12+\log [\mathrm{O} / \mathrm{H}]_{\text {crit }}$ model produces a cumulative redshift distribution similar to the $\Psi(z) \propto$ $(1+z)^{0.5}$ model discussed in Section 3. Third, we show the effects of a low value of $12+\log [\mathrm{O} / \mathrm{H}]_{\text {crit }}=8$. While the fraction of star formation occurring in systems with $12+\log [\mathrm{O} / \mathrm{H}]<8$ is much smaller than $12+\log [\mathrm{O} / \mathrm{H}] \approx 8.7$, normalized over the redshift range $0<z<4$ the redshift dependence of the two models is similar. Sensibly, the low $12+\log [\mathrm{O} / \mathrm{H}]_{\text {crit }}$ model evolves with a somewhat stronger redshift dependence as the epoch at where the characteristic stellar mass in the stellar mass function reaches $12+\log [\mathrm{O} / \mathrm{H}]_{\text {crit }}$ is pushed to higher redshift. This low $12+\log [\mathrm{O} / \mathrm{H}]_{\text {crit }}$ model only serves as a strawman to illustrate the calculated redshift dependence; GRBs are observed to occur at larger metallicities (see, e.g., Levesque et al. 2010a; Mannucci et al. 2011).

As in Section 3, we can formalize this comparison using a one-sample K-S test. Figure 4 shows the K-S test probability as a function of $12+\log [\mathrm{O} / \mathrm{H}]_{\text {crit }}$ in terms of $12+\log [\mathrm{O} / \mathrm{H}]$ on the Kobulnicky \& Kewley (2004) scale for $8 \leqslant 12+$ 


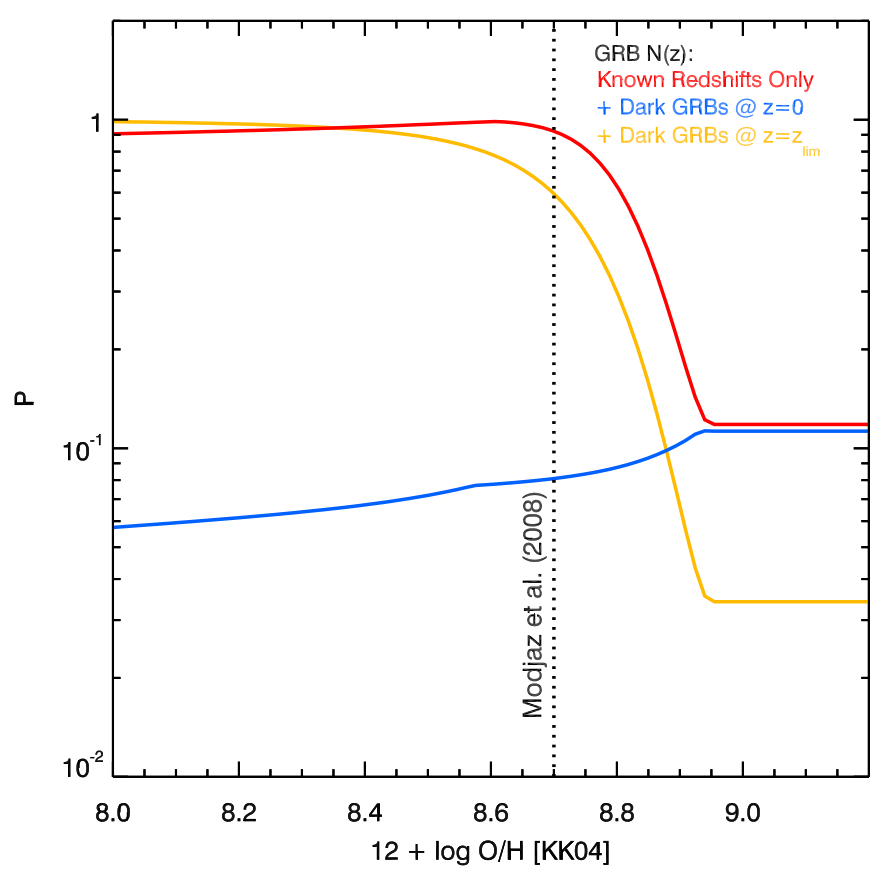

Figure 4. Kolmogorov-Smirnov test results which compare the observed GRB cumulative redshift distribution with the calculated distribution $N\left(<z \mid z_{\max }=4\right)$ assuming the Hopkins \& Beacom (2006) model for the star formation rate density $\dot{\rho}_{\star}(z)$ with a redshift-dependent GRB rate to SFR ratio $\Psi(z)$ given by Equation (7). Shown is the probability $P$ of consistency as a function of the critical metallicity $12+\log [\mathrm{O} / \mathrm{H}]_{\text {crit }}$ (on the scale of Kobulnicky \& Kewley 2004) above which GRBs are suppressed. For the sample with firm redshifts (red line) all critical metallicities produce K-S $P>0.05$, meaning the data are consistent with no metallicity ceiling (note that at $12+\log [\mathrm{O} / \mathrm{H}]_{\text {crit }} \gtrsim 9$ the models are equivalent to $\alpha=0$ in Figure 2). However, the peak probability is very similar to the metallicity ceiling claimed by Modjaz et al. (2008) (dotted line) and the consistency of the model and data rapidly increases for $12+\log [\mathrm{O} / \mathrm{H}]_{\text {crit }}<8.9$. Including the dark GRB sample assuming they lie at their maximum possible redshift shifts the $P>0.05$ interval to $12+\log [\mathrm{O} / \mathrm{H}]_{\text {crit }}<8.9$ (formally, this interval equates to a $2 \sigma$ Gaussian equivalent confidence; see the text). If dark GRBs were all local $(z \approx 0$, blue line), no clear value for $12+\log [\mathrm{O} / \mathrm{H}]_{\text {crit }}$ is favored.

(A color version of this figure is available in the online journal.)

$\log [\mathrm{O} / \mathrm{H}]_{\text {crit }} \leqslant 9.2$. We find that a cumulative GRB redshift distribution produced by a $12+\log [\mathrm{O} / \mathrm{H}]_{\text {crit }} \lesssim 8.85$ produces a redshift evolution in $\Psi(z)$ that is adequately consistent $(P>$ 0.05 ) with the firm GRB sample (red line) or the sample enlarged by dark GRBs at their redshift limits (orange line). Using Equations (1) and (7) to construct the likelihood function $\mathcal{L}\left(12+\log [\mathrm{O} / \mathrm{H}]_{\text {crit }}\right)$ and the posterior distribution $p(12+$ $\left.\log [\mathrm{O} / \mathrm{H}]_{\text {crit }} \mid \mathbf{z}, \Psi\right)$, we find that the firm GRB redshift sample is formally $2 \sigma$ consistent with no metallicity ceiling. Similarly, we find that including the dark GRB sample at their redshift limits has a $2 \sigma$ confidence interval of $12+\log [\mathrm{O} / \mathrm{H}]_{\text {crit }}<8.9$. We note that in both these models the maximum probability occurs for a metallicity similar to the ceiling $12+\log [\mathrm{O} / \mathrm{H}]_{\text {crit }} \approx 8.7$ suggested by Modjaz et al. (2008, Figure 4 dotted line). While the cumulative GRB distribution prefers a $12+\log [\mathrm{O} / \mathrm{H}]_{\text {crit }} \lesssim$ 8.7, lower critical metallicities display similar consistency. We note that these low critical metallicities can in principle be differentiated based on absolute comparisons of the GRB rate, rather than through normalized cumulative distribution functions. Finally, if dark GRBs are local $(z \sim 0)$ phenomena (blue line), then the observed GRB distribution is not very constraining with a large range in $12+\log [\mathrm{O} / \mathrm{H}]_{\text {crit }}$ displaying similar consistency with the data.
In summary, it is relatively straightforward to construct physically plausible models for $\Psi(z)$ within the sample uncertainties, based on a metallicity ceiling for GRB production. As the sample sizes grow, there is every prospect of securing valuable constraints on such models. As a note, we caution that the recent observations by Savaglio et al. (2011) of the afterglow of GRB 090323 at redshift $z \sim 4$ show evidence for two damped Lyman$\alpha$ s (DLAs) with supersolar metallicities, and that Cenko et al. (2011) suggest at least one of these DLAs may be associated with the GRB host galaxy. If these observations are confirmed, they could pose difficulty for models of the GRB rate with a low $12+\log [\mathrm{O} / \mathrm{H}]_{\text {crit }}$.

\section{THE HIGH-REDSHIFT STAR FORMATION RATE DENSITY DERIVED FROM DISTANT GRBs}

An exciting development in the past few years has been the discovery and verification of the first sample of longduration GRBs beyond redshifts $z \simeq 6$ (e.g., Kawai et al. 2006; Tanvir et al. 2009; Salvaterra et al. 2009a; Cucchiara et al. 2011). We now utilize our understanding of the ratio of the GRB rate to the SFR density $\Psi$ and its possible redshift dependence to interpret these data. Of particular interest is how such GRB-derived estimates of the SFR density $\dot{\rho}_{\star}(z)$ compare with those determined from UV-selected galaxy samples, as these quantities hold implications for the timing of cosmic reionization and whether the density of star-forming galaxies alone provides sufficient energetic radiation to reionize the IGM. In the following we will follow closely the procedures and arguments developed in Robertson et al. (2010).

In the manner of Yüksel et al. (2008), we can estimate the SFR density as

$$
\left\langle\dot{\rho}_{\star}\right\rangle\left(z_{1}, z_{2}\right)=\frac{N_{\mathrm{GRB}}\left(z_{1}, z_{2}\right)}{N_{\mathrm{GRB}}(1,4)} \frac{\int_{1}^{4} \dot{\rho}_{\star}(z) \Psi(z) \frac{d V}{d z} \frac{d z}{1+z}}{\int_{z_{1}}^{z_{2}} \Psi(z) \frac{d V}{d z} \frac{d z}{1+z}},
$$

where $N_{\mathrm{GRB}}\left(z_{1}, z_{2}\right)$ is the observed number of GRBs at redshifts $z_{1}<z<z_{2}$. Panel (a) of Figure 5 shows the resulting comparison. The SFR density determined from UV-selected galaxies (McLure et al. 2010; Bouwens et al. 2007, 2010a; Oesch et al. 2010; Schiminovich et al. 2005; Reddy \& Steidel 2009) increases to $z \sim 3$ and then declines to high redshift (Bouwens et al. 2011b, gray points with error bars). Two parameterized star formation histories from Robertson et al. (2010) consistent with the data are shown for illustration (green hatched area). SFR densities estimated from the high-redshift $(z>4)$ GRB rate as calculated by Equation (16) and three models from Figures 1 and 3 are shown as red $\left(\alpha=0\right.$, equivalent to $\left.12+\log [\mathrm{O} / \mathrm{H}]_{\text {crit }} \sim 9\right)$, blue $\left(12+\log [\mathrm{O} / \mathrm{H}]_{\text {crit }} \approx 8.7\right.$, equivalent to $\alpha \approx 0.5$ at $z \lesssim 4$ and $\alpha \approx 0$ at $z \gtrsim 4)$, and orange points $\left(\Psi(z) \propto(1+z)^{1.5}\right.$, stronger evolution than any metallicity evolution model studied in Section 4.1). All models have Poisson error bars indicated. The four lower- $z$ points at $z=4.25,4.75,5.25$, and 6.75 contain $N=7,3,6$, and 4 GRBs, respectively. The highest redshift bin $(z \approx 8.75)$ contains the two highest-redshift GRBs observed $(z=8.2$, Tanvir et al. 2009; Salvaterra et al. 2009a; $z=9.4$, Cucchiara et al. 2011).

Clearly the SFR densities estimated from the high-redshift GRB rate through Equation (16) for physically plausible models are considerably higher than those inferred from UV-selected galaxies. The model GRB rate to SFR ratio calculated in Section 4 has a redshift dependence that is constant at $\psi \sim 1$ above $z \gtrsim 4$, and the GRB-inferred $\dot{\rho}_{\star}(z)$ at high redshift 

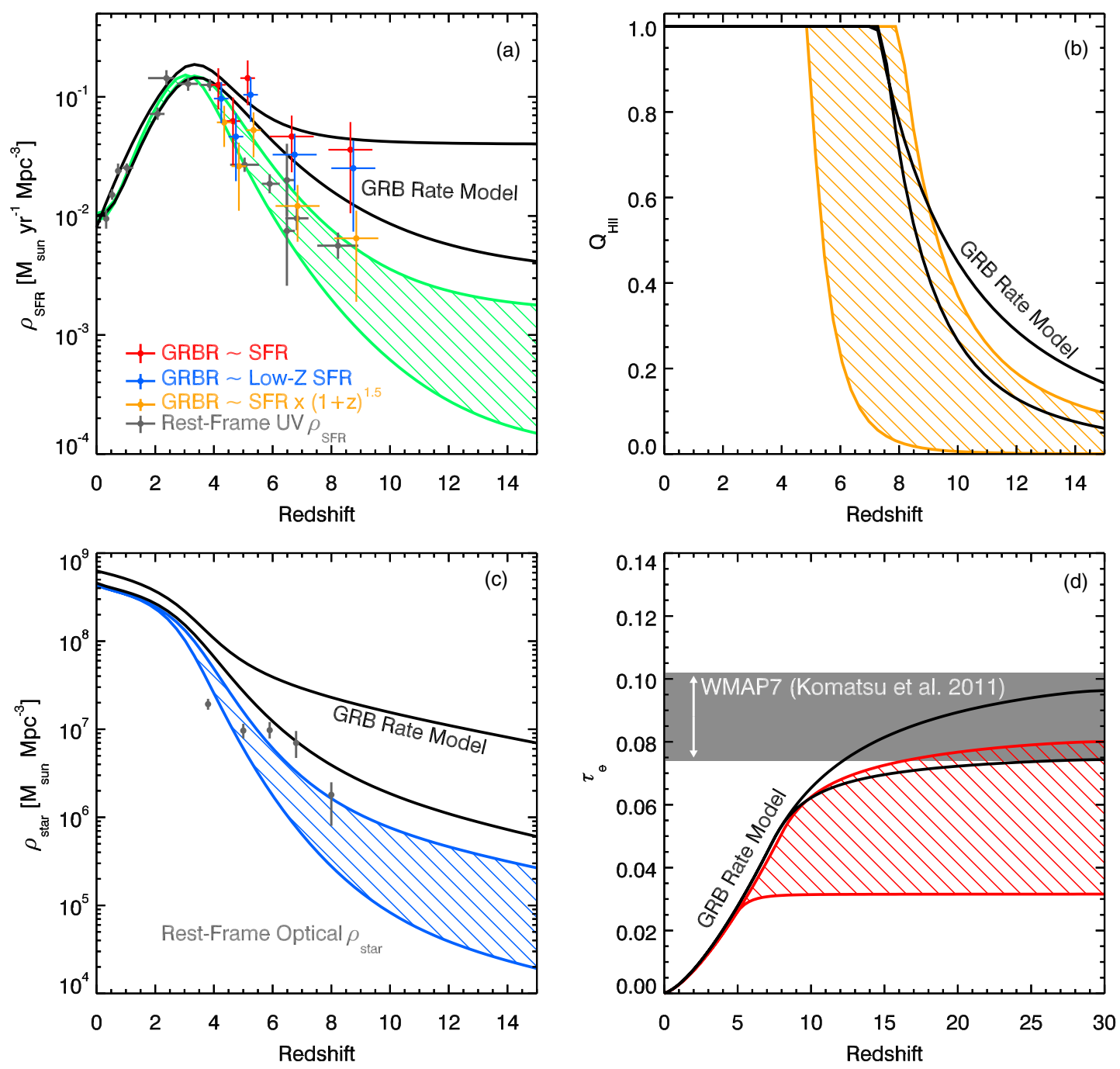

Figure 5. Implications of GRB-derived estimates for the high-redshift star formation rate density, $\dot{\rho}_{\star}(z)$. Panel (a) (upper left) shows $\dot{\rho}_{\star}(z)$ determined from UV-selected galaxies (gray points with error bars, see the text) and corresponding parametric SFR histories from Robertson et al. (2010) (green hatched region). Also shown are values implied by the GRB rate assuming no evolution in $\Psi(z)$ (red points), our model for GRB production in low-metallicity galaxies (blue points), and strong evolution in GRB production per unit star formation rate $\left(\Psi(z) \propto(1+z)^{1.5}\right.$, orange points). The model points have been offset slightly in redshift for clarity and the model error bars reflect Poisson errors on the GRB rate in each redshift bin. If the GRB rate to SFR ratio evolves weakly beyond $z>4$ (red and blue points), the rate of discovery of high-redshift GRBs already implies a $\dot{\rho}_{\star}(z)$ much larger than that inferred from UV-selected galaxies. Evolution faster than $\Psi(z) \propto(1+z)^{1.5}$ would be needed to force agreement. Parameterized star formation histories consistent with the GRB-derived star formation histories in the constant $\Psi$ and low metallicity star formation models are shown as black lines. With fiducial choices about the character of the stellar populations $\left(Z \sim 0.2 Z_{\odot}\right)$, the clumpiness of the intergalactic medium ( $C=3$, upper line; $C=2.5$ lower line), and the escape fraction of ionizing photons ( $f_{\text {esc }}=0.06$, upper line; $f_{\text {esc }}=0.2$, lower line) we can calculate the reionization history in panel (b) (upper right) implied by the GRB-derived high-redshift star formation rate (black lines) and compare with similar histories calculated by Robertson et al. (2010) determined from UV-selected galaxies (orange hatched region). The GRB-inferred star formation history would produce a large volume filling fraction of ionized gas extending to high redshift. The path length through this ionized gas to the cosmic microwave background provides the optical depth to electron scattering $\tau_{e}$ in panel (d) (lower right). The ionization history computed from the GRB-derived star formation history would easily reach $\tau_{e}$ implied by the seven-year Wilkinson Microwave Anisotropy Probe measurements (Komatsu et al. 2011) and produce a much larger value than that similarly calculated from UV-selected galaxies (red hatched area; Robertson et al. 2010). While both the ionization history and the Thomson optical depth depend on specific model choices for $f_{\text {esc }}$ or $C$, the stellar mass density (panel (c), lower left) is simply determined by the integral of the previous star formation rate density (panel (a), upper left). The stellar mass density to $z \sim 8$ is shown as gray points with error bars (González et al. 2011), with the associated models by Robertson et al. (2010, blue hatched region). The black lines in panel (c) show the stellar mass density implied by parameterizations of the GRB-derived star formation rate, which clearly exceed the stellar mass density at all redshifts.

(A color version of this figure is available in the online journal.)

is therefore large in this model. By considering the recently discovered GRBs at the highest redshifts (Tanvir et al. 2009; Salvaterra et al. 2009a; Cucchiara et al. 2011), we have extended this result to $z \sim 9.5$. The results are in contrast to recent claims for a low abundance of $z \gtrsim 10$ star-forming galaxies (e.g., Bouwens et al. 2011a; Oesch et al. 2011). To reconcile $\dot{\rho}_{\star}(z)$ estimates from both GRBs and UV-selected galaxies would require a dramatic evolution in $\Psi(z)$. The physical basis for such an evolution is unclear (cf. Section 4).

\section{DISCUSSION: IMPLICATION FOR COSMIC REIONIZATION}

As the discovery of high-redshift galaxies (McLure et al. 2010; Bouwens et al. 2010a; Oesch et al. 2010) and quasars (Mortlock et al. 2011) reaches beyond $z>7$, it is important to understand the potential role for star-forming galaxies in reionizaton (for a review, see Robertson et al. 2010). For instance, Robertson et al. (2010) have calculated the reionization 
history, Thomson scattering optical depth, and stellar mass build-up for the star formation histories plotted in Figure 5 (hatched regions). The star formation histories in these models have been parameterized using the formula

$$
\dot{\rho}_{\star}(z)=\frac{a+b(z / c)^{f}}{1+(z / c)^{d}}+g,
$$

which is the Robertson et al. (2010) generalization of the model by Cole et al. (2001) to include a floor in the SFR. In Figure 5, panel (a), the upper star formation history of the green hatched area has parameter values $a=0.009 M_{\odot} \mathrm{yr}^{-1} \mathrm{Mpc}^{-3}$, $b=0.27 M_{\odot} \mathrm{yr}^{-1} \mathrm{Mpc}^{-3}, c=3.7, d=7.4$, and $g=$ $10^{-3} M_{\odot} \mathrm{yr}^{-1} \mathrm{Mpc}^{-3}$. The lower star formation history model has $c=3.4, d=8.3$, and $g=10^{-4} M_{\odot} \mathrm{yr}^{-1} \mathrm{Mpc}^{-3}$. With metal-poor stellar populations, a typical escape fraction of $f_{\text {esc }} \sim 0.3$ and a clumping factor $C \sim 2$, the upper $\dot{\rho}_{\star}(z)$ curve fully ionizes the IGM by $z \sim 7$ and recovers the Wilkinson Microwave Anisotropy Probe (WMAP) electron scattering optical measurement (e.g., Komatsu et al. 2011).

Using the same form of Equation (17) to parameterize the SFR density implied by the high-redshift GRB rate and a weak-to-moderate redshift dependence of $\Psi(z)$ (Figure 5, panel (a), red and blue points), we find that parameter values of $a=0.007 M_{\odot} \mathrm{yr}^{-1} \mathrm{Mpc}^{-3}, b=0.27 M_{\odot} \mathrm{yr}^{-1} \mathrm{Mpc}^{-3}$, $c=3.7, d=6.4, f=2.5$, and $g=3 \times 10^{-3} M_{\odot} \mathrm{yr}^{-1} \mathrm{Mpc}^{-3}$ are representative as a "low- $\dot{\rho}_{\star}(z)$ )" GRB-derived model (lower black curve $)$, while adopting $d=7.4$ and $g=\left[\left(4 \times 10^{-2}-\right.\right.$ $\left.\left.10^{-3}\right) \times(z / 3)+10^{-3}\right] M_{\odot} \mathrm{yr}^{-1} \mathrm{Mpc}^{-3}$ provides representative "high- $\dot{\rho}_{\star}(z)$ " GRB-derived model (upper black curve).

With the same assumptions for the escape fraction, IGM clumping factor, and stellar population model used by Robertson et al. (2010), the star formation history implied by the GRB rate for either a constant $\Psi(z)$ or an evolution of the GRB rate to SFR ratio $\Psi(z)$ that tracks star formation in low-metallicity galaxies would fully reionize the universe by $z \sim 14$ and significantly overpredict the Thomson scattering optical depth (e.g., $\tau \sim 0.2$ ). If instead high-redshift galaxies have metallicities $Z \sim 0.2 Z_{\odot}$ with a Schaerer (2003) stellar population, the escape fraction is lower $\left(f_{\text {esc }} \sim 0.06\right)$, and the clumping factor is $C \sim 3$, the high- $\dot{\rho}_{\star}(z)$ model implied by the GRB rate induces reionization by $z \sim 7$ and produces a Thomson scattering optical depth which can match the WMAP value (upper black curve in Figure 5, panels (b) and (d)). For $f_{\text {esc }}=0.2$ and $C=3$, the low- $\dot{\rho}_{\star}(z)$ GRB-derived model produces some what lower ionized gas volume filling factors and Thomson optical depths (lower black curve in Figure 5). Models by Wyithe et al. (2010) reach similar conclusions.

Although the uncertainties associated with calculating $\dot{\rho}_{\star}(z)$ from GRBs are large, an explanation of the possible discrepancy between the GRB-inferred $\dot{\rho}_{\star}(z)$ and the abundance of highredshift galaxies is warranted. The higher values deduced for the GRB-inferred $\dot{\rho}_{\star}(z)$ compared to those inferred from UV-selected galaxies may strengthen the case for a steep luminosity function for the latter and hence closure on the hypothesis that intrinsically faint star-forming galaxies over $6<z<12$ were responsible for cosmic reionization (e.g., Yüksel et al. 2008; Kistler et al. 2009). However, as emphasized by Robertson et al. (2010), there is a further constraint provided by the observed stellar mass density at $z \simeq 5-6$. This constraint is determined from rest-frame optical fluxes deduced from the Infrared Array Camera (IRAC) instrument on board Spitzer Space Telescope. Importantly, the $\dot{\rho}_{\star}(z)$ implied by the highredshift GRB rate appears unphysical in that it overproduces the observed stellar mass density at $z \gtrsim 5$. Panel (c) of Figure 5 shows the rest-frame optical stellar mass density determinations by González et al. (2011, gray points with error bars; see also Eyles et al. 2005; Stark et al. 2007, 2009; Labbé et al. 2006, 2010; and González et al. 2010). These data may be compared with the integral of the parameterized SFR densities consistent by the high-redshift GRB-derived $\dot{\rho}_{\star}(z)$ (Figure 5, panel (c), black lines). While the escape fraction, clumping factor, and stellar population properties are not well known and may be sensibly varied within the broad uncertainties to match the observed Thomson optical depth to electron scattering, simultaneously accounting for the comparably low observed stellar mass density at high redshift is difficult. While deeper IRAC observations and other techniques are improving these constraints (V. Gonzalez et al., in preparation) it is important to recognize that these new estimates have confirmed that previous efforts have properly accounted for incompleteness (e.g., Stark et al. 2007) and the stellar mass density measures are unlikely to increase substantially owing to future data.

We thus conclude there is an important conflict between fairly reasonable assumptions about how the GRB rate maps to cosmic star formation and what we already understand about early star formation from UV-selected galaxies. Can the two probes of early star formation be reconciled? Dust is an unlikely explanation given we would require all UV-selected star-forming galaxies to be heavily extincted and most studies have utilized their UV continuum slopes to infer the absence of any significant reddening (Bouwens et al. 2010b; Finkelstein et al. 2010; McLure et al. 2011; Dunlop et al. 2011). The highredshift UV galaxy luminosity functions used to determine the SFR density data in Figure 5 are taken from Bouwens et al. (2011b). At $z=8$ the Bouwens et al. (2011b) luminosity function has a measured faint end slope of $\gamma=-2$, and the luminosity density at $z=8$ shown in Figure 5 corresponds to a limiting absolute magnitude of $M_{\mathrm{AB}}=-18$. It has been suggested that extending the search to much fainter sources will help bridge the gap between the galaxy- and GRB-inferred $\dot{\rho}_{\star}(z)$ (see Figure 4 of Kistler et al. 2009 and Choi \& Nagamine 2011), but even with the very steep $\gamma=-2$ the luminosity function would need to continue down to $M_{\mathrm{AB}}=-(7-9)$ - slightly brighter than the globular cluster scale-for the UV-luminosity density to recover the GRB-inferred SFR density. Deeper Hubble Space Telescope (HST) data will clarify the possibility. Adjusting the early stellar initial mass function (IMF) so as to increase the luminosity output per unit $\dot{\rho}_{\star}(z)$ would reduce the stellar mass density; for a Salpeter (1955) IMF with an upper mass limit of $M_{\max }=100 M_{\odot}$, the minimum mass of forming stars would have to increase from $M_{\min } \approx 0.1 M_{\odot}$ to $M_{\min } \approx 2.75-4 M_{\odot}$ to decrease the long-lived stellar mass by the required factor of $f_{\star} \sim 4-5$. Conceivably, the IRAC fluxes at $z \simeq 5-6$ are significantly contaminated by nebular emission so that the deduced stellar masses are overestimated. An adjustment to the IMF could provide as much as $f_{\star}^{2}$ times as much nebular emission contamination in the IRAC bands compared to the nebular emission expected from a standard Salpeter (1955) stellar population. In this case, one factor of $f_{\star}$ increase in the nebular emission contribution to the IRAC flux would arise from the increase in the number of Lyman continuum photons produced per unit star formation, and another factor of $f_{\star}$ would arise from the decrease in the contribution of long-lived stars to the rest-frame optical emission relative to a Salpeter (1955) IMF. Luminosity evolution in the GRB population could also contribute by altering $\Psi(z)$ (e.g., 
Lloyd-Ronning et al. 2002; Firmani et al. 2004; Kocevski \& Liang 2006; Salvaterra \& Chincarini 2007; Salvaterra et al. 2009b; Virgili et al. 2011), but this is also uncertain (e.g., Butler et al. 2010; Wanderman \& Piran 2010). More likely, there are additional physical factors affecting the high-redshift GRB production rate, i.e., beyond those of star formation and simple metallicity dependence considered in this paper. Possible examples include the physics of angular momentum retention in GRB progenitors (e.g., MacFadyen et al. 2001) or the effects of the cosmic background radiation temperature on the initial mass function (e.g., Larson 1998, 2005). Regardless of what the cause might be, it is clear that the continued discovery and study of $z>6 \mathrm{GRBs}$ promise to shed light on the reionization process and the assumptions made in the interpretation of SFRs and stellar masses from UV-selected galaxies.

\section{SUMMARY}

Using the second Swift BAT catalog of GRBs (Sakamoto et al. 2011), the observations of dark GRBs by Perley et al. (2009), Greiner et al. (2011), and Krühler et al. (2011) and the GRB catalogs of Butler et al. (2007, 2010), we have constructed the cumulative redshift distribution $N\left(<z \mid z_{\max }\right)$ of 112 luminous $\left(L_{\text {iso }}>10^{51} \mathrm{erg} \mathrm{s}^{-1}\right)$ GRBs out to redshift $z \sim 9$. Comparing with models of an evolving GRB rate to SFR ratio $\Psi(z)$, we find that $N\left(<z \mid z_{\max }\right)$ constrains redshift evolution of the form $\Psi(z) \propto(1+z)^{\alpha}$ to $0 \lesssim \alpha \lesssim 1.5$ out to $z_{\max } \approx 4$. By extending the model of Kocevski et al. (2009) to calculate $\Psi(z)$ from the evolution of the mass-metallicity relation (Savaglio et al. 2005), the star-formation-mass relation (Drory \& Alvarez 2008), and the stellar mass function (Drory et al. 2005), we find that the presence of a host galaxy metallicity ceiling $12+\log [\mathrm{O} / \mathrm{H}]_{\text {crit }}$ above which GRBs are suppressed is highly consistent with the available data if $12+\log [\mathrm{O} / \mathrm{H}]_{\text {crit }} \lesssim 8.85$. The peak probability of consistency between the model and data occurs at $12+\log [\mathrm{O} / \mathrm{H}]_{\text {crit }} \sim 8.7$, near the GRB metallicity ceiling sug- gested by Modjaz et al. (2008). Using the method of Yüksel et al. (2008) and Kistler et al. (2009), we use the GRB rate at $1<z<4$ (where the SFR density $\dot{\rho}_{\star}(z)$ is roughly constant) to estimate the $\dot{\rho}_{\star}(z)$ at $z>4$ (including constraints from the highest redshift GRBs, e.g., Kawai et al. 2006; Tanvir et al. 2009; Salvaterra et al. 2009a; Cucchiara et al. 2011). We find that for constant to moderate $(\alpha<1)$ redshift evolution in $\Psi(z)$, the SFR density predicted by the observed high-redshift GRB rate is substantially higher than the observed $\dot{\rho}_{\star}(z)$ inferred from the abundance of UV-selected galaxies (e.g., Bouwens et al. 2010a; McLure et al. 2010), and would overproduce the observed high-redshift stellar mass density (e.g., Stark et al. 2009; González et al. 2011). Rough agreement between the UV- and GRB-determined $\dot{\rho}_{\star}(z)$ can be achieved if the redshift dependence of $\Psi(z)$ is as strong as $\alpha \gtrsim 1.5$ at $z \gtrsim 4$.

We thank the anonymous referee for useful suggestions that improved the manuscript. We also thank Maryam Modjaz and Ehud Nakar for helpful comments and discussions. B.E.R. was supported by a Hubble Fellowship grant program number HST-HF-51262.01-A provided by NASA from the Space Telescope Science Institute, which is operated by the Association of Universities for Research in Astronomy, Incorporated, under NASA contract NAS5-26555.

\section{APPENDIX \\ GRB SAMPLE}

Our analysis relies heavily on the excellent GRB catalogs provided by Butler et al. (2007), Butler et al. (2010), and the Second Swift BAT catalog (Sakamoto et al. 2011). Further, we make use of the important studies by Perley et al. (2009), Greiner et al. (2011), and Krühler et al. (2011) that provided redshift constraints for a sample of dark GRBs. Below in Table 1, we provide the union of these catalogs (162 GRBs in total) as

Table 1

GRB Catalog

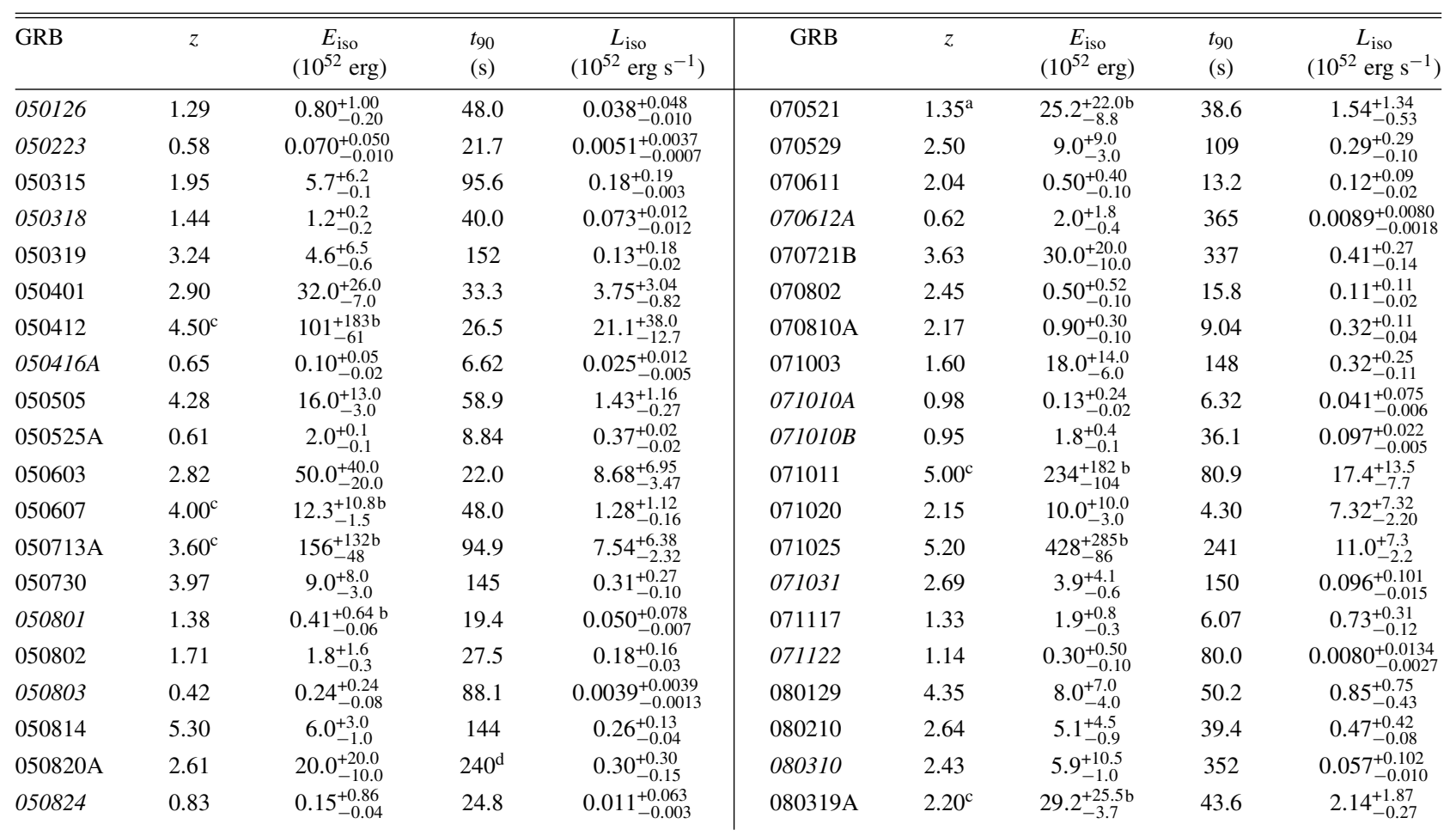


Table 1

(Continued)

\begin{tabular}{|c|c|c|c|c|c|c|c|c|c|}
\hline GRB & $z$ & $\begin{array}{c}E_{\text {iso }} \\
\left(10^{52} \mathrm{erg}\right)\end{array}$ & $\begin{array}{l}t_{90} \\
(\mathrm{~s})\end{array}$ & $\begin{array}{c}L_{\text {iso }} \\
\left(10^{52} \mathrm{erg} \mathrm{s}^{-1}\right)\end{array}$ & GRB & $z$ & $\begin{array}{c}E_{\text {iso }} \\
\left(10^{52} \mathrm{erg}\right)\end{array}$ & $\begin{array}{l}t_{90} \\
(\mathrm{~s})\end{array}$ & $\begin{array}{c}L_{\text {iso }} \\
\left(10^{52} \mathrm{erg} \mathrm{s}^{-1}\right)\end{array}$ \\
\hline 050826 & 0.30 & $0.03_{-0.02}^{+0.04}$ & 35.7 & $0.0011_{-0.0007}^{+0.0015}$ & 080319B & 0.94 & $400_{-100}^{+200}$ & 125 & $6.21_{-1.55}^{+3.10}$ \\
\hline 050904 & 6.29 & $130_{-40}^{+70}$ & 182 & $5.22_{-1.61}^{+2.81}$ & 080319C & 1.95 & $6.0_{-1.0}^{+5.0}$ & 29.5 & $0.60_{-0.10}^{+0.50}$ \\
\hline 050908 & 3.35 & $1.3_{-0.3}^{+0.9}$ & 18.3 & $0.31_{-0.07}^{+0.21}$ & 080320 & $7.00^{\mathrm{c}}$ & $34.2_{-5.7}^{+45.6 \mathrm{~b}}$ & 13.8 & $19.8_{-3.3}^{+26.4}$ \\
\hline $050915 A$ & $0.40^{\mathrm{a}}$ & $0.073_{-0.023}^{+0.102 b}$ & 53.4 & $0.0019_{-0.0006}^{+0.0027}$ & 080330 & 1.51 & $0.41_{-0.06}^{+0.94}$ & 67.1 & $0.015_{-0.002}^{+0.035}$ \\
\hline 050922C & 2.20 & $3.9_{-0.8}^{+2.7}$ & 4.54 & $2.75_{-0.56}^{+1.90}$ & 080411 & 1.03 & $23.0_{-4.0}^{+0.9}$ & 56.3 & $0.83_{-0.14}^{+0.03}$ \\
\hline $051016 B$ & 0.94 & $0.037_{-0.006}^{+0.056}$ & 4.02 & $0.018_{-0.003}^{+0.027}$ & 080413A & 2.43 & $9.0_{-2.0}^{+6.0}$ & 46.4 & $0.67_{-0.15}^{+0.44}$ \\
\hline 051109A & 2.35 & $2.3_{-0.5}^{+2.4}$ & 37.2 & $0.21_{-0.04}^{+0.22}$ & 080413B & 1.10 & $1.5_{-0.2}^{+0.2}$ & 8.00 & $0.39_{-0.05}^{+0.05}$ \\
\hline $051109 B$ & 0.08 & $0.00036_{-0.00009}^{+0.00019}$ & 13.4 & $0.00003_{-0.00001}^{+0.00002}$ & 080430 & 0.77 & $0.38_{-0.08}^{+0.30}$ & 14.2 & $0.047_{-0.010}^{+0.037}$ \\
\hline 051111 & 1.55 & $6.0_{-2.0}^{+5.0}$ & 64.0 & $0.24_{-0.08}^{+0.20}$ & 080516 & $3.60^{\mathrm{e}}$ & $12.0_{-4.8}^{+6.0 \mathrm{~b}}$ & 5.75 & $\begin{array}{l}9.57_{-3.83}^{+4.79} \\
\text {. }\end{array}$ \\
\hline 060108 & 2.03 & $0.59_{-0.08}^{+0.84}$ & 14.2 & $0.13_{-0.02}^{+0.18}$ & 080520 & 1.55 & $0.11_{-0.04}^{+1.45}$ & 3.32 & $0.084_{-0.031}^{+1.112}$ \\
\hline 060110 & $5.00^{\mathrm{c}}$ & $83.3_{-15.6}^{+67.7 \mathrm{~b}}$ & 21.1 & $23.6_{-4.4}^{+19.2}$ & 080603B & 2.69 & $6.0_{-1.0}^{+1.0}$ & 59.1 & $0.37_{-0.06}^{+0.06}$ \\
\hline 060115 & 3.53 & $6.0_{-1.0}^{+2.0}$ & 122 & $0.22_{-0.04}^{+0.07}$ & 080604 & 1.42 & $0.70_{-0.10}^{+0.80}$ & 69.2 & $0.024_{-0.003}^{+0.028}$ \\
\hline 060116 & 6.60 & $21.0_{-7.0}^{+16.0}$ & 105 & $1.52_{-0.51}^{+1.16}$ & 080605 & 1.64 & $21.0_{-4.0}^{+9.0}$ & 18.0 & $3.07_{-0.59}^{+1.32}$ \\
\hline 060124 & 2.30 & $0.70_{-0.10}^{+0.70}$ & 658 & $0.0035_{-0.0005}^{+0.0035}$ & 080607 & 3.04 & $280_{-90}^{+130}$ & 78.9 & $14.3_{-4.6}^{+6.7}$ \\
\hline 060202 & 0.78 & $0.70_{-0.10}^{+0.60}$ & 172 & $0.0073_{-0.0010}^{+0.0062}$ & 080707 & 1.23 & $0.34_{-0.05}^{+0.41}$ & 30.2 & $0.025_{-0.004}^{+0.030}$ \\
\hline 060206 & 4.06 & $4.1_{-0.7}^{+1.2}$ & 7.55 & $2.75_{-0.47}^{+0.80}$ & 080710 & 0.85 & $0.80_{-0.40}^{+0.80}$ & 112 & $0.013_{-0.007}^{+0.013}$ \\
\hline 060210 & 3.91 & $42.0_{-8.0}^{+35.0}$ & 242 & $0.85_{-0.16}^{+0.71}$ & 080721 & 2.59 & $110_{-50}^{+110}$ & 176 & $2.24_{-1.02}^{+2.24}$ \\
\hline 060218 & 0.03 & $0.00029_{-0.00007}^{+0.00014}$ & $128^{\mathrm{d}}$ & $2.3 \times 10^{-6}$ & 080804 & 2.20 & $16.0_{-7.0}^{+17.0}$ & 37.2 & $1.38_{-0.60}^{+1.46}$ \\
\hline 060223A & 4.41 & $3.1_{-0.5}^{+1.2}$ & 11.3 & $1.48_{-0.24}^{+0.57}$ & 080805 & 1.50 & $4.0_{-2.0}^{+2.0}$ & 107 & $0.094_{-0.047}^{+0.047}$ \\
\hline 060418 & 1.49 & $10.0_{-2.0}^{+7.0}$ & 109 & $0.23_{-0.05}^{+0.16}$ & 080810 & 3.36 & $30.0_{-20.0}^{+20.0}$ & 108 & $1.21_{-0.81}^{+0.81}$ \\
\hline $060428 B$ & 0.35 & $0.020_{-0.004}^{+0.019}$ & 96.0 & $0.00028_{-0.00006}^{+0.00027}$ & 080905B & 2.37 & $3.4_{-0.6}^{+3.1}$ & 102 & $0.11_{-0.02}^{+0.10}$ \\
\hline 060502A & 1.50 & $3.2_{-0.9}^{+2.8}$ & 28.5 & $0.28_{-0.08}^{+0.25}$ & 080913 & 6.70 & $7.0_{-1.0}^{+7.0}$ & 7.46 & $7.23_{-1.03}^{+7.23}$ \\
\hline 060510B & 4.94 & $23.0_{-4.0}^{+10.0}$ & 263 & $0.52_{-0.09}^{+0.23}$ & 080916A & 0.69 & $0.81_{-0.09}^{+0.21}$ & 61.3 & $0.022_{-0.002}^{+0.006}$ \\
\hline 060512 & 2.10 & $0.81_{-0.16}^{+0.16 f}$ & 11.4 & $0.22_{-0.04}^{+0.04}$ & 080928 & 1.69 & $2.8_{-0.5}^{+2.4}$ & 234 & $0.032_{-0.006}^{+0.028}$ \\
\hline 060522 & 5.11 & $7.0_{-1.0}^{+7.0}$ & 69.1 & $0.62_{-0.09}^{+0.62}$ & 081007 & 0.53 & $0.07_{-0.01}^{+0.05}$ & 9.01 & $0.012_{-0.002}^{+0.008}$ \\
\hline 060526 & 3.22 & $5.2_{-0.4}^{+5.6}$ & 275 & $0.080_{-0.006}^{+0.086}$ & 081008 & 1.97 & $6.0_{-1.0}^{+3.0}$ & 180 & $0.099_{-0.017}^{+0.050}$ \\
\hline 060604 & 2.68 & $0.50_{-0.10}^{+1.20}$ & 96.0 & $0.019_{-0.004}^{+0.046}$ & 081028 & 3.04 & $11.0_{-2.0}^{+3.0}$ & 284 & $0.16_{-0.03}^{+0.04}$ \\
\hline 060605 & 3.77 & $2.5_{-0.6}^{+3.1}$ & 539 & $0.022_{-0.005}^{+0.027}$ & 081029 & 3.85 & $15.0_{-7.0}^{+9.0}$ & 275 & $0.26_{-0.12}^{+0.16}$ \\
\hline 060607A & 3.07 & $9.0_{-2.0}^{+7.0}$ & 103 & $0.36_{-0.08}^{+0.28}$ & 081109 & $0.98^{\mathrm{g}}$ & $4.1_{-2.2}^{+2.6 \mathrm{~b}}$ & 221 & $0.037_{-0.020}^{+0.023}$ \\
\hline 060614 & 0.13 & $0.24_{-0.04}^{+0.04}$ & 109 & $0.0025_{-0.0004}^{+0.0004}$ & 081118 & 2.58 & $2.8_{-0.4}^{+4.4}$ & 49.3 & $0.20_{-0.03}^{+0.32}$ \\
\hline 060707 & 3.42 & $6.1_{-0.9}^{+1.9}$ & 66.7 & $0.40_{-0.06}^{+0.13}$ & 081121 & 2.51 & $16.0_{-4.0}^{+15.0}$ & 17.7 & $3.18_{-0.80}^{+2.98}$ \\
\hline 060708 & 1.92 & $0.60_{-0.10}^{+0.40}$ & 9.96 & $0.18_{-0.03}^{+0.12}$ & 081203A & 2.05 & $17.0_{-4.0}^{+13.0}$ & 223 & $0.23_{-0.05}^{+0.18}$ \\
\hline 060714 & 2.71 & $7.7_{-0.9}^{+7.5}$ & 116 & $0.25_{-0.03}^{+0.24}$ & 081222 & 2.77 & $15.0_{-2.0}^{+3.0}$ & 33.0 & $1.71_{-0.23}^{+0.34}$ \\
\hline 060729 & 0.54 & $0.33_{-0.06}^{+0.29}$ & 113 & $0.0045_{-0.0008}^{+0.0040}$ & 081228 & $3.40^{\mathrm{e}}$ & $3.7_{-1.3}^{+1.6 \mathrm{~b}}$ & 3.00 & $5.36_{-1.84}^{+2.30}$ \\
\hline 060805A & $3.80^{\mathrm{c}}$ & $1.8_{-0.5}^{+2.7 \mathrm{~b}}$ & 4.93 & $1.72_{-0.53}^{+2.65}$ & 090102 & 1.55 & $14.0_{-5.0}^{+10.0}$ & 29.3 & $1.22_{-0.43}^{+0.87}$ \\
\hline 060814 & 0.84 & $11.6_{-2.0}^{+5.8 b}$ & 145 & $0.15_{-0.03}^{+0.07}$ & 090205 & 4.65 & $1.2_{-0.2}^{+1.6}$ & 8.80 & $0.77_{-0.13}^{+1.03}$ \\
\hline $060904 B$ & 0.70 & $0.30_{-0.06}^{+0.19}$ & 172 & $0.0030_{-0.0006}^{+0.0019}$ & 090313 & 3.38 & $4.6_{-0.5}^{+7.0}$ & 70.7 & $0.28_{-0.03}^{+0.43}$ \\
\hline 060906 & 3.69 & $13.0_{-1.0}^{+12.0}$ & 44.6 & $1.37_{-0.11}^{+1.26}$ & $090417 B$ & 0.34 & $0.16_{-0.03}^{+0.16 \mathrm{~b}}$ & 282 & $0.00074_{-0.00015}^{+0.00076}$ \\
\hline 060908 & 1.88 & $8.1_{-4.5}^{+1.9 \mathrm{~h}}$ & 18.8 & $1.24_{-0.69}^{+0.29}$ & 090418A & 1.61 & $9.0_{-3.0}^{+6.0}$ & 56.3 & $0.42_{-0.14}^{+0.28}$ \\
\hline 060912A & 0.94 & $0.80_{-0.20}^{+0.50}$ & 5.03 & $0.31_{-0.08}^{+0.19}$ & 090423 & 8.23 & $8.0_{-1.0}^{+2.0}$ & 9.77 & $7.56_{-0.94}^{+1.89}$ \\
\hline 060923A & $4.00^{\mathrm{c}}$ & $20.0_{-4.6}^{+9.2 \mathrm{~b}}$ & 51.5 & $1.94_{-0.45}^{+0.89}$ & 090424 & 0.54 & $2.6_{-0.4}^{+0.4}$ & 49.5 & $0.081_{-0.012}^{+0.012}$ \\
\hline 060926 & 3.21 & $1.0_{-0.2}^{+2.2}$ & 7.79 & $0.54_{-0.11}^{+1.19}$ & 090429B & 9.40 & $54.9_{-7.8}^{+15.7 b}$ & 5.61 & $101.7_{-14.5}^{+29.1}$ \\
\hline 060927 & 5.46 & $9.0_{-1.0}^{+2.0}$ & 22.4 & $2.59_{-0.29}^{+0.58}$ & 090510 & 0.90 & $0.30_{-0.20}^{+0.50}$ & 5.66 & $0.10_{-0.07}^{+0.17}$ \\
\hline 061004 & 3.30 & $2.0_{-0.3}^{+1.1}$ & 6.26 & $1.37_{-0.21}^{+0.76}$ & 090516 & 4.11 & $50.0_{-10.0}^{+50.0}$ & 208 & $1.23_{-0.25}^{+1.23}$ \\
\hline 061007 & 1.26 & $140_{-60}^{+110}$ & 75.7 & $4.18_{-1.79}^{+3.29}$ & 090519 & 3.85 & $15.0_{-8.0}^{+13.0}$ & 58.3 & $1.25_{-0.67}^{+1.08}$ \\
\hline 061021 & 0.35 & $0.40_{-0.16}^{+0.32 \mathrm{~b}}$ & 43.8 & $0.012_{-0.005}^{+0.010}$ & 090529A & 2.62 & $2.5_{-0.5}^{+1.4}$ & $80.0^{\mathrm{d}}$ & $0.11_{-0.02}^{+0.06}$ \\
\hline 061110A & 0.76 & $0.28_{-0.06}^{+0.28}$ & 44.5 & $0.011_{-0.002}^{+0.011}$ & 090618 & 0.54 & $15.0_{-1.0}^{+1.0}$ & 113 & $0.20_{-0.01}^{+0.01}$ \\
\hline 061110B & 3.43 & $13.0_{-6.0}^{+16.0}$ & 133 & $0.43_{-0.20}^{+0.53}$ & 090715B & 3.00 & $24.0_{-5.0}^{+15.0}$ & 265 & $0.36_{-0.08}^{+0.23}$ \\
\hline 061121 & 1.31 & $19.0_{-5.0}^{+11.0}$ & 81.2 & $0.54_{-0.14}^{+0.31}$ & 090726 & 2.71 & $1.8_{-0.4}^{+2.1}$ & 56.7 & $0.12_{-0.03}^{+0.14}$ \\
\hline 061126 & 1.16 & $8.0_{-2.0}^{+7.0}$ & 50.3 & $0.34_{-0.09}^{+0.30}$ & 090809A & 2.74 & $1.4_{-0.4}^{+2.4}$ & 7.84 & $0.67_{-0.19}^{+1.14}$ \\
\hline 061222A & 2.09 & $67.4_{-12.8}^{+35.3 b}$ & 96.0 & $2.17_{-0.41}^{+1.14}$ & 090812 & 2.45 & $19.0_{-5.0}^{+17.0}$ & 75.1 & $0.87_{-0.23}^{+0.78}$ \\
\hline
\end{tabular}


Table 1

(Continued)

\begin{tabular}{lcccc|ccccc}
\hline \hline GRB & $z$ & $\begin{array}{c}E_{\text {iso }} \\
\left(10^{52} \mathrm{erg}\right)\end{array}$ & $\begin{array}{c}t_{90} \\
(\mathrm{~s})\end{array}$ & $\begin{array}{c}L_{\text {iso }} \\
\left(10^{52} \mathrm{erg} \mathrm{s}^{-1}\right)\end{array}$ & GRB & $z$ & $\begin{array}{c}E_{\text {iso }} \\
\left(10^{52} \mathrm{erg}\right)\end{array}$ & $\begin{array}{c}t_{90} \\
(\mathrm{~s})\end{array}$ & $\begin{array}{c}L_{\text {iso }} \\
\left(10^{52} \mathrm{erg} \mathrm{s}^{-1}\right)\end{array}$ \\
\hline $061222 \mathrm{~B}$ & 3.35 & $8.0_{-2.0}^{+7.0}$ & 37.2 & $0.94_{-0.23}^{+0.82}$ & $090814 A$ & 0.70 & $0.27_{-0.03}^{+0.03 \mathrm{f}}$ & 78.2 & $0.0059_{-0.0007}^{+0.0007}$ \\
070110 & 2.35 & $43.0_{-0.5}^{+2.5}$ & 79.7 & $1.81_{-0.02}^{+0.11}$ & $090904 \mathrm{~B}$ & $5.00^{\mathrm{i}}$ & $34.4_{-14.0}^{+14.0 \mathrm{f}}$ & 64.0 & $3.23_{-1.31}^{+1.31}$ \\
070208 & 1.17 & $0.28_{-0.08}^{+0.22}$ & 64.0 & $0.0095_{-0.0027}^{+0.0074}$ & $090926 \mathrm{~B}$ & 1.24 & $5.4_{-2.0}^{+2.8 \mathrm{~h}}$ & 99.3 & $0.12_{-0.05}^{+0.06}$ \\
070306 & 1.50 & $6.0_{-1.0}^{+5.0}$ & 209 & $0.072_{-0.012}^{+0.060}$ & 090927 & 1.37 & $0.23_{-0.03}^{+0.03 \mathrm{f}}$ & 2.16 & $0.25_{-0.03}^{+0.03}$ \\
070318 & 0.84 & $0.90_{-2.00}^{+0.90}$ & 132 & $0.013_{-0.028}^{+0.013}$ & 091018 & 0.97 & $0.70_{-0.10}^{+0.30 \mathrm{~h}}$ & 4.37 & $0.32_{-0.05}^{+0.14}$ \\
070411 & 2.95 & $10.0_{-2.0}^{+8.0}$ & 102 & $0.39_{-0.08}^{+0.31}$ & 091020 & 1.71 & $7.5_{-0.3}^{+0.3 \mathrm{f}}$ & 38.9 & $0.52_{-0.02}^{+0.02}$ \\
$070419 \mathrm{~A}$ & 0.97 & $0.24_{-0.05}^{+0.23}$ & 160 & $0.0030_{-0.0000}^{+0.0028}$ & 091024 & 1.09 & $3.9_{-0.2}^{+0.2 \mathrm{f}}$ & 112 & $0.072_{-0.003}^{+0.003}$ \\
070506 & 2.31 & $0.26_{-0.05}^{+0.17}$ & 4.35 & $0.20_{-0.04}^{+0.13}$ & 091029 & 2.75 & $8.5_{-2.5}^{+4.5 \mathrm{~h}}$ & 39.2 & $0.81_{-0.24}^{+0.43}$ \\
070508 & 0.82 & $8.0_{-1.0}^{+2.0}$ & 20.9 & $0.70_{-0.09}^{+0.17}$ & 091127 & 0.49 & $0.77_{-0.02}^{+0.02 \mathrm{f}}$ & 7.42 & $0.16_{-0.00}^{+0.00}$ \\
070518 & 1.16 & $0.090_{-0.010}^{+0.150}$ & 4.35 & $0.045_{-0.005}^{+0.074}$ & $091208 \mathrm{~B}$ & 1.06 & $1.9_{-0.1}^{+0.1 \mathrm{f}}$ & 14.8 & $0.27_{-0.02}^{+0.02}$ \\
\hline
\end{tabular}

Notes.

${ }^{\text {a }}$ Redshift from Perley et al. (2009).

${ }^{\mathrm{b}} E_{\text {iso }}$ calculated from fluence provided by Butler et al. $(2007,2010)$.

${ }^{c}$ Dark GRB redshift limit from Perley et al. (2009).

${ }^{\mathrm{d}}$ Burst duration taken from Butler et al. $(2007,2010)$.

${ }^{\mathrm{e}}$ Redshift from Greiner et al. (2011).

${ }^{\mathrm{f}} E_{\text {iso }}$ calculated from fluence provided by Table 2 of Sakamoto et al. (2011).

${ }^{\mathrm{g}}$ Redshift from Krühler et al. (2011).

${ }^{\mathrm{h}} E_{\text {iso }}$ from Table 13 of Sakamoto et al. (2011).

${ }^{i}$ Dark GRB redshift limit from Greiner et al. (2011).

a convenience to the reader only. The papers providing these catalogs should be consulted for important details on the fluence measurements and redshift determinations, along with further original works discovering and characterizing individual GRBs. The table presents redshifts, isotropic equivalent energies and luminosities, and burst durations as described in Section 2.1. Of these quantities, only the isotropic-equivalent energies for 29 GRBs (noted below) and the luminosities (for all GRBs) are newly computed in this work. Most of the $E_{\text {iso }}$ values reported (129) are taken from Butler et al. (2007, 2010), with four recent $E_{\text {iso }}$ values adopted from Sakamoto et al. (2011, noted below). Of the 29 GRBs whose energies we compute as $E_{\text {iso }}=4 \pi d_{L}^{2} S$, where $d_{L}$ is the luminosity distance to a redshift $z$ and $S$ is the fluence, 21 broad-band fluences are taken from Butler et al. (2007, 2010) and 8 10-150 keV fluences (effectively lower limits) are taken from Sakamoto et al. (2011). These GRBs are also noted in the table. Dark GRB redshifts (indicated as upper limits where appropriate and noted) are adopted from Perley et al. (2009), Greiner et al. (2011), or Krühler et al. (2011). Their reported energies and luminosities are calculated assuming that the GRBs lie at the redshift upper limits. Burst durations mostly are taken from Table 1 of Sakamoto et al. (2011), except for three values taken from Butler et al. (2007, 2010, noted in table). For completeness reasons our analysis makes use of luminous $\left(L_{\text {iso }}>10^{51} \mathrm{erg} \mathrm{s}^{-1}\right)$ GRBs (see Section 2.1), and less luminous GRBs are therefore indicated with italicized names.

\section{REFERENCES}

Amati, L., Frontera, F., Tavani, M., et al. 2002, A\&A, 390, 81 Arnouts, S., Schiminovich, D., Ilbert, O., et al. 2005, ApJ, 619, L43 Baldry, I. K., \& Glazebrook, K. 2003, ApJ, 593, 258

Bouwens, R., Broadhurst, T., \& Illingworth, G. 2003a, ApJ, 593, 640 Bouwens, R., \& Illingworth, G. 2006, New Astron. Rev., 50, 152 Bouwens, R. J., Illingworth, G. D., Franx, M., \& Ford, H. 2007, ApJ, 670, 928 Bouwens, R. J., Illingworth, G. D., Labbe, I., et al. 2011a, Nature, 469, 504 Bouwens, R. J., Illingworth, G. D., Oesch, P. A., et al. 2010a, ApJ, 709, L133 Bouwens, R. J., Illingworth, G. D., Oesch, P. A., et al. 2010b, ApJ, 708, L69 Bouwens, R. J., Illingworth, G. D., Oesch, P. A., et al. 2011b, ApJ, 737, 90
Bouwens, R. J., Illingworth, G. D., Rosati, P., et al. 2003b, ApJ, 595, 589 Bromm, V., \& Loeb, A. 2002, ApJ, 575, 111

Bunker, A. J., Stanway, E. R., Ellis, R. S., \& McMahon, R. G. 2004, MNRAS, 355,374

Butler, N. R., Bloom, J. S., \& Poznanski, D. 2010, ApJ, 711, 495

Butler, N. R., Kocevski, D., Bloom, J. S., \& Curtis, J. L. 2007, ApJ, 671, 656

Cenko, S. B., Frail, D. A., Harrison, F. A., et al. 2011, ApJ, 732, 29

Chary, R., Berger, E., \& Cowie, L. 2007, ApJ, 671, 272

Choi, J.-H., \& Nagamine, K. 2011, MNRAS, in press

Cole, S., Norberg, P., Baugh, C. M., et al. 2001, MNRAS, 326, 255

Cucchiara, A., Levan, A. J., Fox, D. B., et al. 2011, ApJ, 736, 7

Dai, X. 2009, ApJ, 697, L68

Drory, N., \& Alvarez, M. 2008, ApJ, 680, 41

Drory, N., Salvato, M., Gabasch, A., et al. 2005, ApJ, 619, L131

Dunlop, J. S., McLure, R. J., Robertson, B. E., et al. 2011, arXiv:1102.5005

Erb, D. K., Shapley, A. E., Pettini, M., et al. 2006, ApJ, 644, 813

Eyles, L. P., Bunker, A. J., Stanway, E. R., et al. 2005, MNRAS, 364, 443

Finkelstein, S. L., Papovich, C., Giavalisco, M., et al. 2010, ApJ, 719, 1250

Firmani, C., Avila-Reese, V., Ghisellini, G., \& Tutukov, A. V. 2004, ApJ, 611, 1033

Fontana, A., Vanzella, E., Pentericci, L., et al. 2010, ApJ, 725, L205

Fruchter, A. S., Levan, A. J., Strolger, L., et al. 2006, Nature, 441, 463

Fruchter, A. S., Thorsett, S. E., Metzger, Mark R., et al. 1999, ApJ, 519, L13

Fryer, C. L., Woosley, S. E., \& Hartmann, D. H. 1999, ApJ, 526, 152

Fynbo, J. P. U., Jakobsson, P., Möller, P., et al. 2003, A\&A, 406, L63

Fynbo, J. P. U., Jakobsson, P., Prochaska, J. X., et al. 2009, ApJS, 185, 526

Galama, T. J., Vreeswijk, P. M., van Paradijs, J., et al. 1998, Nature, 395, 670

Gehrels, N., Chincarini, G., Giommi, P., et al. 2004, ApJ, 611, 1005

González, V., Labbé, I., Bouwens, R. J., et al. 2010, ApJ, 713, 115

González, V., Labbé, I., Bouwens, R. J., et al. 2011, ApJ, 735, L34

Greiner, J., Krühler, T., Fynbo, J. P. U., et al. 2009, ApJ, 693, 1610

Greiner, J., Krühler, T., Klose, S., et al. 2011, A\&A, 526, A30

Han, X. H., Hammer, F., Liang, Y. C., et al. 2010, A\&A, 514, A24

Hanish, D. J., Meurer, G. R., Ferguson, H. C., et al. 2006, ApJ, 649, 150

Hirschi, R., Meynet, G., \& Maeder, A. 2005, A\&A, 443, 581

Hjorth, J., Sollerman, J., Møller, P., et al. 2003, Nature, 423, 847

Hopkins, A. M. 2004, ApJ, 615, 209

Hopkins, A. M., \& Beacom, J. F. 2006, ApJ, 651, 142

Hopkins, A. M., \& Beacom, J. F. 2008, ApJ, 682, 1486

Ishida, E. E. O., de Souza, R. S., \& Ferrara, A. 2011, MNRAS, 418, 500

Iye, M., Ota, K., Kashikawa, N., et al. 2006, Nature, 443, 186

Kawai, N., Kosugi, G., Aoki, K., et al. 2006, Nature, 440, 184

Kewley, L. J., \& Ellison, S. L. 2008, ApJ, 681, 1183

Kistler, M. D., Yüksel, H., Beacom, J. F., Hopkins, A. M., \& Wyithe, J. S. B. 2009, ApJ, 705, L104 
Kistler, M. D., Yüksel, H., Beacom, J. F., \& Stanek, K. Z. 2008, ApJ, 673, L119 Kobulnicky, H. A., \& Kewley, L. J. 2004, ApJ, 617, 240

Kocevski, D., \& Liang, E. 2006, ApJ, 642, 371

Kocevski, D., West, A. A., \& Modjaz, M. 2009, ApJ, 702, 377

Komatsu, E., Smith, K. M., Dunkley, J., et al. 2011, ApJS, 192, 18

Krühler, T., et al. 2011, A\&A, 534, 108

Labbé, I., Bouwens, R., Illingworth, G. D., \& Franx, M. 2006, ApJ, 649, L67

Labbé, I., González, V., Bouwens, R. J., et al. 2010, ApJ, 708, L26

Langer, N., \& Norman, C. A. 2006, ApJ, 638, L63

Larson, R. B. 1998, MNRAS, 301, 569

Larson, R. B. 2005, MNRAS, 359, 211

Le Floc'h, E., Duc, P.-A., Mirabel, I. F., et al. 2003, A\&A, 400, 499

Le Floc'h, E., Papovich, C., Dole, H., et al. 2005, ApJ, 632, 169

Lehnert, M. D., Nesvadba, N. P. H., Cuby, J.-G., et al. 2010, Nature, 467, 940

Levesque, E. M., Kewley, L. J., Berger, E., \& Zahid, H. J. 2010a, AJ, 140, 1557

Levesque, E. M., Kewley, L. J., Graham, J. F., \& Fruchter, A. S. 2010b, ApJ, 712, L26

Levesque, E. M., Soderberg, A. M., Foley, R. J., et al. 2010c, ApJ, 709, L26 Lloyd-Ronning, N. M., Fryer, C. L., \& Ramirez-Ruiz, E. 2002, ApJ, 574, 554

MacFadyen, A. I., Woosley, S. E., \& Heger, A. 2001, ApJ, 550, 410

Mannucci, F., Cresci, G., Maiolino, R., Marconi, A., \& Gnerucci, A. 2010, MNRAS, 408, 2115

Mannucci, F., Salvaterra, R., \& Campisi, M. A. 2011, MNRAS, 414, 1263

Mao, S., \& Mo, H. J. 1998, A\&A, 339, L1

McLure, R. J., Dunlop, J. S., Cirasuolo, M., et al. 2010, MNRAS, 403, 960

McLure, R. J., et al. 2011, MNRAS, in press

Mészáros, P. 2006, Rep. Prog. Phys., 69, 2259

Modjaz, M. 2011, Astron. Nachr., 332, 434

Modjaz, M., Kewley, L., Kirshner, R. P., et al. 2008, AJ, 135, 1136

Modjaz, M., Stanek, K. Z., Garnavich, P. M., et al. 2006, ApJ, 645, L21

Mortlock, D. J., Warren, S. J., Venemans, B. P., et al. 2011, Nature, 474, 616

Oesch, P. A., Bouwens, R. J., Illingworth, G. D., et al. 2010, ApJ, 709, L16

Oesch, P. A., et al. 2011, arXiv:1105.2297

Ono, Y., et al. 2011, arXiv:1107.3159

Ouchi, M., Shimasaku, K., Okamura, S., et al. 2004, ApJ, 611, 660

Pentericci, L., Fontana, A., Vanzella, E., et al. 2011, ApJ, 743, 132

Pérez-González, P. G., Rieke, G. H., Egami, E., et al. 2005, ApJ, 630, 82

Perley, D. A., Cenko, S. B., Bloom, J. S., et al. 2009, AJ, 138, 1690

Porciani, C., \& Madau, P. 2001, ApJ, 548, 522

Prochaska, J. X., Bloom, J. S., Chen, H.-W., et al. 2004, ApJ, 611, 200

Reddy, N. A., \& Steidel, C. C. 2009, ApJ, 692, 778

Robertson, B. E., Ellis, R. S., Dunlop, J. S., McLure, R. J., \& Stark, D. P. 2010, Nature, 468, 49
Sakamoto, T., Barthelmy, S. D., Baumgartner, W. H., et al. 2011, ApJS, 195,2

Salpeter, E. E. 1955, ApJ, 121, 161

Salvaterra, R., \& Chincarini, G. 2007, ApJ, 656, L49

Salvaterra, R., Della Valle, M., Campana, S., et al. 2009a, Nature, 461, 1258

Salvaterra, R., Guidorzi, C., Campana, S., Chincarini, G., \& Tagliaferri, G. 2009b, MNRAS, 396, 299

Savaglio, S., Glazebrook, K., Le Borgne, D., et al. 2005, ApJ, 635, 260

Savaglio, S., Glazebrook, K., \& Le Borgne, D. 2009, ApJ, 691, 182

Savaglio, S., et al. 2011, arXiv:1110.4642

Schaerer, D. 2003, A\&A, 397, 527

Schechter, P. 1976, ApJ, 203, 297

Schenker, M. A., Stark, D. P., Ellis, R. S., et al. 2011, arXiv:1107.1261

Schiminovich, D., Ilbert, O., Arnouts, S., et al. 2005, ApJ, 619, L47

Soderberg, A. M., Chakraborti, S., Pignata, G., et al. 2010, Nature, 463, 513

Sollerman, J., Östlin, G., Fynbo, J. P. U., et al. 2005, New Astron., 11, 103

Stanek, K. Z., Gnedin, O. Y., Beacom, J. F., et al. 2006, Acta Astron., 56, 333

Stanek, K. Z., Matheson, T., Garnavich, P. M., et al. 2003, ApJ, 591, L17

Stark, D. P., Bunker, A. J., Ellis, R. S., Eyles, L. P., \& Lacy, M. 2007, ApJ, 659 84

Stark, D. P., Ellis, R. S., Bunker, A., et al. 2009, ApJ, 697, 1493

Stark, D. P., Ellis, R. S., Chiu, K., Ouchi, M., \& Bunker, A. 2010, MNRAS, 408, 1628

Tanvir, N. R., Fox, D. B., Levan, A. J., et al. 2009, Nature, 461, 1254

Thompson, R. I., Eisenstein, D., Fan, X., et al. 2006, ApJ, 647, 787

Totani, T. 1997, ApJ, 486, L71

Tremonti, C. A., Heckman, T. M., Kauffmann, G., et al. 2004, ApJ, 613, 898

Vanzella, E., Pentericci, L., Fontana, A., et al. 2011, ApJ, 730, L35

Virgili, F. J., Zhang, B., Nagamine, K., \& Choi, J.-H. 2011, MNRAS, 417, 3025

Wanderman, D., \& Piran, T. 2010, MNRAS, 406, 1944

Wiersema, K., Savaglio, S., Vreeswijk, P. M., et al. 2007, A\&A, 464, 529

Wijers, R. A. M. J., Bloom, J. S., Bagla, J. S., \& Natarajan, P. 1998, MNRAS, 294, L13

Wolf, C., Meisenheimer, K., Rix, H.-W., et al. 2003, A\&A, 401, 73

Woosley, S. E. 1993, ApJ, 405, 273

Woosley, S. E., \& Bloom, J. S. 2006, ARA\&A, 44, 507

Woosley, S. E., \& Heger, A. 2006, ApJ, 637, 914

Wyithe, J. S. B., Hopkins, A. M., Kistler, M. D., Yüksel, H., \& Beacom, J. F. 2010, MNRAS, 401, 2561

Yoon, S.-C., \& Langer, N. 2005, A\&A, 443, 643

Yüksel, H., Kistler, M. D., Beacom, J. F., \& Hopkins, A. M. 2008, ApJ, 683, L5 